\title{
Hadronic and partonic sources of direct photons in relativistic heavy-ion collisions
}

\author{
O. Linnyk * V. Konchakovski, T. Steinert, and W. Cassing \\ Institut für Theoretische Physik, Universität Giessen, 35392 Giessen, Germany \\ E. L. Bratkovskaya \\ Institut für Theoretische Physik, Johann Wolfgang Goethe University, 60438 Frankfurt am Main, \\ Germany; Frankfurt Institute for Advanced Studies, 60438 Frankfurt am Main, Germany;
}

(Dated: August 7, 2018)

\begin{abstract}
The direct photon spectra and flow $\left(v_{2}, v_{3}\right)$ in heavy-ion collisions at SPS, RHIC and LHC energies are investigated within a relativistic transport approach incorporating both hadronic and partonic phases - the Parton-Hadron-String Dynamics (PHSD). In the present work, four extensions are introduced compared to our previous calculations: (i) going beyond the soft-photon approximation (SPA) in the calculation of the bremsstrahlung processes meson + meson $\rightarrow$ meson + meson $+\gamma$, (ii) quantifying the suppression due to the Landau-Pomeranchuk-Migdal (LPM) coherence effect, (iii) adding the additional channels $V+N \rightarrow N+\gamma$ and $\Delta \rightarrow N+\gamma$ and (iv) providing PHSD calculations for $\mathrm{Pb}+\mathrm{Pb}$ collisions at $\sqrt{s_{N N}}=2.76 \mathrm{TeV}$. The first issue extends the applicability of the bremsstrahlung calculations to higher photon energies in order to understand the relevant sources in the region $p_{T}=0.5-1.5 \mathrm{GeV}$, while the LPM correction turns out to be important for $p_{T}<0.4 \mathrm{GeV}$ in the partonic phase. The results suggest that a large elliptic flow $v_{2}$ of the direct photons signals a significant contribution of photons produced in interactions of secondary mesons and baryons in the late (hadronic) stage of the heavy-ion collision. In order to further differentiate the origin of the direct photon azimuthal asymmetry (late hadron interactions vs electromagnetic fields in the initial stage), we provide predictions for the photon spectra, elliptic flow and triangular flow $v_{3}\left(p_{T}\right)$ of direct photons at different centralities to be tested by the experimental measurements at the LHC energies. Additionally, we illustrate the magnitude of the photon production in the partonic and hadronic phases as functions of time and local energy density. Finally, the 'cocktail' method for an estimation of the background photon elliptic flow, which is widely used in the experimental works, is supported by the calculations within the PHSD transport approach.
\end{abstract}

PACS numbers: 25.75.-q, 13.60.Le, 14.40.Lb, 14.65.Dw

\section{INTRODUCTION}

Direct photons are a powerful probe of the quarkgluon plasma (QGP) as created in ultra-relativistic nuclear collisions. The photons interact only electromagnetically and thus escape to the detector almost undistorted through the dense and strongly-interacting medium. Thus the photon transverse-momentum spectra and their azimuthal asymmetry carry information on the properties of the matter under extreme conditions, existing in the first few $\mathrm{fm} / \mathrm{c}$ of the collisional evolution.

On the other hand, the measured photons provide a time-integrated picture of the heavy-ion collision dynamics and are emitted from every moving charge - partons or hadrons. Therefore, a multitude of photon sources has to be differentiated in order to access the signal of interest. The dominant contributions to the inclusive photon production are the decays of mesons, dominantly pions, $\eta$ - and $\omega$-mesons. Experimental collaborations subtract the "decay photons" from the inclusive photon spectrum using a cocktail calculation [1, 2] and obtain the "direct" photons.

In particular the direct photons at transverse momenta

*Electronic address: Olena.Linnyk@theo.physik.uni-giessen.de
$\left(p_{T}<3 \mathrm{GeV}\right)$ are expected to be dominated by "thermal" sources, i.e. the radiation from the strongly interacting Quark-Gluon-Plasma (sQGP) [3] and the secondary meson+meson and meson+baryon interactions in the hadronic phase [4, 5]. These partonic and hadronic channels have been studied within PHSD in detail in Refs. [6, 7] at Relativistic-Heavy-Ion-Collider (RHIC) energies. It was found that the partonic channels constitute up to half of the observed direct photon spectrum for very central collisions. Other theoretical calculations also find a significant contribution of the photons produced in the QGP to the direct photon spectrum [8 13].

The low- $p_{T}$ direct photons probe not only the temperature [1, 2, 13] of the produced QCD-matter, but also its (transport) properties, for instance, the shear viscosity. Using the direct photon elliptic flow $v_{2}$ (a measure of the azimuthal asymmetry in the photon distribution) as a viscosimeter was first suggested by Dusling et al. in Ref. [14]; this idea was later supported by the calculations in Refs. 10, 11, 13, 15]. It was also suggested that the photon spectra and $v_{2}$ are sensitive to the collective directed flow of the system [16, 17], to the equation of state [16, 18], to the possible production of a Glasma 19 21 , to the rate of chemical equilibration in the QGP [2224] and to the asymmetry induced by the strong magnetic field (flash) in the very early stage of the collision [25 28].

However, the recent observation by the PHENIX Col- 
laboration [1] that the elliptic flow $v_{2}\left(p_{T}\right)$ of 'direct photons' produced in minimum bias $\mathrm{Au}+\mathrm{Au}$ collisions at $\sqrt{s_{N N}}=200 \mathrm{GeV}$ is comparable to that of the produced pions was a surprise and in contrast to the theoretical expectations and predictions. Indeed, the photons produced by partonic interactions in the quark-gluon plasma phase have not been expected to show considerable flow because they are dominated by the emission in the initial phase before the elliptic flow fully develops.

In Refs. [6, 7] we have applied the PHSD approach to photon production in $\mathrm{Au}+\mathrm{Au}$ collisions at $\sqrt{s_{N N}}=$ $200 \mathrm{GeV}$ and studied the transverse momentum spectrum and the elliptic flow $v_{2}$ of photons from hadronic and partonic production channels. The microscopic description of the full collision evolution is calculated in the covariant off-shell transport approach PHSD. The degrees-of-freedom in the partonic and hadronic phases are strongly interacting dynamical quasi-particles and off-shell hadrons, respectively.

It was found in Refs. [6, 7] that the PHSD calculations reproduce the transverse momentum spectrum of direct photons as measured by the PHENIX Collaboration in Refs. [29, 30]. The centrality dependence of the thermal photon yield in PHSD was predicted to be $\sim N_{\text {part }}^{\alpha}$ with the exponent $\alpha=1.5$, which is in good agreement with the most recent measurement of $\alpha=1.48 \pm 0.08 \pm 0.04$ by the PHENIX Collaboration 31]. Also, the PHSD described the data on the elliptic flow of inclusive and direct photons at the top RHIC energy. The strong $v_{2}$ of direct photons - which is comparable to the hadronic $v_{2}$ - in PHSD was attributed to hadronic channels, i.e. to meson binary reactions which are not subtracted in the data. As sources for photon production, we have incorporated the interactions of off-shell quarks and gluons in the strongly interacting quark-gluon plasma (sQGP) $(q+\bar{q} \rightarrow g+\gamma$ and $q(\bar{q})+g \rightarrow q(\bar{q})+\gamma)$, the decays of hadrons $(\pi \rightarrow \gamma+\gamma$, $\left.\eta \rightarrow \gamma+\gamma, \omega \rightarrow \pi+\gamma, \eta^{\prime} \rightarrow \rho+\gamma, \phi \rightarrow \eta+\gamma, a_{1} \rightarrow \pi+\gamma\right)$ as well as their interactions $\pi+\pi \rightarrow \rho+\gamma, \rho+\pi \rightarrow \pi+\gamma$, meson-meson bremsstrahlung $m+m \rightarrow m+m+\gamma$ ), meson-baryon bremsstrahlung $(m+B \rightarrow m+B+\gamma)$. In the present work we additionally incorporate the two-totwo vector meson+nucleon interactions $(V+p \rightarrow \gamma+p / n$ and $V+n \rightarrow \gamma+p / n)$ and the decay of the $\Delta$-resonance $\Delta \rightarrow N \gamma$.

The photon production via bremsstrahlung in mesonmeson and meson-baryon elastic collisions was found to be a very important source to interpret the data on the direct photon spectra and elliptic flow simultaneously [6, 7]. In the previous works [ 6, , 7], we have been calculating the photon bremsstrahlung from all elastic meson-meson and meson-baryon scatterings $m_{1}+m_{2}$, which occur during the heavy-ion collisions (including $\left.m_{i}=\pi, \eta, K, \bar{K}, K^{0}, K^{*}, \bar{K}^{*}, K^{* 0}, \eta^{\prime}, \omega, \rho, \phi, a_{1}\right)$. For the calculation of the bremsstrahlung cross sections we have been applying the soft-photon approximation (SPA). Therefore the resulting yield of the bremsstrahlung photons depended on model assumptions such as i) the cross section for the meson-meson elastic scattering (we as- sumed $10 \mathrm{mb}$ for all meson species), ii) incoherence of the individual scatterings and iii) the soft-photon approximation (i.e. low photon energy and low $\sqrt{s}$ of the collision). The adequacy of the SPA assumption has been studied before in Ref. 32] and a theoretical uncertainty of up to a factor of 2 was found.

In view of the importance of questions that can be answered by direct photon measurements, we have improved our implementation of the photon production in the PHSD transport approach, in particular of the bremsstrahlung photon production in the meson-meson collisions. In the scope of the present work, we depart from the assumptions above by i) using a covariant oneboson-exchange (OBE) chiral model for the $\pi$ interactions, ii) investigating the suppression due to the coherence of the photon emission with long wavelength (LPM effect) and iii) deriving and implementing the "exact" OBE cross sections for photon bremsstrahlung thus departing from the SPA.

The results of our calculations are compared to the data from the SPS and RHIC Collaborations to check if the earlier conclusions and interpretations hold. Additionally, we provide calculations for the photon production in $P b+P b$ collisions at the energy of $\sqrt{s_{N N}}=$ $2.76 \mathrm{TeV}$ (spectra, elliptic $v_{2}$ and triangular $v_{3}$ flow of direct photons). The comparison of these calculations to the future data of the ALICE Collaboration will be of great interest since the preliminary data [2, 33] indicate a significant direct photon signal at low $p_{T}$ with a large elliptic flow.

\section{PHOTON PRODUCTION IN HEAVY-ION COLLISIONS WITHIN THE PHSD APPROACH}

The PHSD model [34, 35] is an off-shell transport approach that consistently describes the full evolution of a relativistic heavy-ion collision from the initial hard scatterings and string formation through the dynamical deconfinement phase transition to the quark-gluon plasma as well as hadronization and the subsequent interactions in the hadronic phase. The two-particle correlations resulting from the finite width of the parton spectral functions are taken into account dynamically in the PHSD by means of the generalized off-shell transport equations 36] that go beyond the mean field or Boltzmann approximation [37]. The transport theoretical description of quarks and gluons in the PHSD is based on the Dynamical Quasi-Particle Model [38] (DQPM) for partons that is constructed to reproduce lattice QCD (lQCD) results for the entropy density, energy density and pressure as functions of temperature for the quark-gluon plasma in thermodynamic equilibrium. In the hadronic sector, PHSD is equivalent to the Hadron-String Dynamics (HSD) approach [39 41]. For details about the DQPM model and the off-shell transport approach we refer the reader to the review Ref. 37].

We stress that a non-vanishing width in the partonic 
spectral functions is the main difference between the DQPM and conventional quasiparticle models [42]. Its influence on the collision dynamics is essentially seen in the correlation functions. For instance, in the stationary limit, the correlation involving the off-diagonal elements of the energy-momentum tensor $T^{k l}$ defines the shear viscosity $\eta$ of the medium [43]. Here a sizeable width is mandatory to obtain a small ratio of the shear viscosity to entropy density $\eta / s$, which results in a roughly hydrodynamical evolution of the partonic system in PHSD [44]. The finite width leads to two-particle correlations, which are taken into account dynamically by means of the generalized off-shell transport equations [36], going beyond the mean field approximation [37, 45]. It has been shown in [46] that the final width (the imaginary part of the self energy) is demanded by the causality constraint on the propagator in the theory of the strongly interacting particles as soon as the interaction leads to a sizable dressing mass squared (real part of the self energy).

In the past the PHSD approach has provided a consistent description of the bulk properties of heavy-ion collisions - rapidity spectra, transverse mass distributions, azimuthal asymmetries of various particle species - from low Super-Proton-Synchrotron (SPS) up to the LHC energies [34, 35, 47]. In the hadronic sector, PHSD is equivalent to the Hadron-String Dynamics (HSD) approach [39, 40, 48], in which the photon production at top SPS energies has been investigated before in Ref. [41] with an emphasis on the role of meson-meson interactions. The PHSD approach was also successfully used for the analysis of penetrating probes, such as charm [49, 50] and dilepton production from hadronic and partonic sources at SPS, RHIC and LHC energies [51, 52].

Indeed the calculations within the PHSD have reproduced the measured differential spectra of dileptons from heavy-ion collisions at SPS and RHIC energies (see Refs. [51, 52]). Also, it has been checked in Ref. 53] that the dilepton production from the QGP constituents - as incorporated in the PHSD [51, 54] - agrees with the dilepton rate emitted by the thermalized QCD medium as calculated in the 1QCD approach. Indeed, the deconfined state of matter - created in heavy-ion collisions at RHIC [55 58] - was clearly seen in the dilepton yield above invariant masses of $1.2 \mathrm{GeV}$ [51, 52].

As sources of photon production - on top of the general dynamical evolution - we consider hadronic [59] as well as partonic [3, 60] interactions. Let us first describe all the contributions, which consist of the photon production in the quark and gluon collisions, from the hadronic decays and the interactions of intermediate mesons produced throughout the evolution of the nucleus-nucleus collision.

1) Photons are radiated by quarks in the interaction with other quarks and gluons. In this sense, we differentiate two classes of processes: first the two-to-two reactions

$$
\begin{array}{r}
q+\bar{q} \rightarrow g+\gamma, \\
q / \bar{q}+g \rightarrow q / \bar{q}+\gamma .
\end{array}
$$

The implementation of the photon production by the quark and gluon interactions in the PHSD is based on the off-shell cross sections for the interaction of the massive dynamical quasi-particles as described in [,, 54$]$. In addition, photon production in the bremsstrahlung reactions $q+q / g \rightarrow q+q / g+\gamma$ is possible [61].

2) All colliding hadronic charges (meson, baryons) can also radiate photons by the bremsstrahlung processes:

$$
\begin{aligned}
& m+m \rightarrow m+m+\gamma \\
& m+B \rightarrow m+B+\gamma .
\end{aligned}
$$

The processes (1) have been studied within the PHSD in Refs. [6, 41], while the processes (2) were added in Ref. 7]. The implementation of photon bremsstrahlung from hadronic reactions in transport approaches has been based until now on the 'soft photon' approximation (SPA). The soft-photon approximation [62] relies on the assumption that the radiation from internal lines is negligible and the strong interaction vertex is on-shell and is valid only at low energy (and $p_{T}$ ) of the produced photon. Since the relatively high transverse momenta of the direct photons $\left(p_{T}=0.5-1.5 \mathrm{GeV}\right)$ are most important for a potential understanding of the "direct photon puzzle", we extend here the accuracy of our calculations for the photons produced via the bremsstrahlung mechanism beyond the applicability of the SPA. The details of the new model are given below in section IIIA.

3) Additionally, the photons can be produced in binary meson + meson and meson + baryon collisions. We consider here the direct photon production in the following $2 \rightarrow 2$ scattering processes: the meson+meson collisions

$$
\begin{gathered}
\pi+\pi \rightarrow \rho+\gamma, \\
\pi+\rho \rightarrow \pi+\gamma,
\end{gathered}
$$

and the meson+baryon collisions

$$
\begin{gathered}
V+N \rightarrow \gamma+N, \\
\text { where } V=\rho, \phi, \omega, \quad \text { and } N=n, p,
\end{gathered}
$$

accounting for all possible charge combinations. The implementation of the reactions (3) is the same as described in Refs. [6, 41]. On the other hand, the meson+baryon processes (4) are incorporated here for the first time in a transport approach. We describe the relevant cross sections in section IIIC

4) Photon production in the decays of mesons $\left(\pi^{0}, \eta, \eta^{\prime}, \omega, \phi, a_{1}\right)$ and the $\Delta$-resonance, where the parent hadrons are produced in baryon-baryon $(B B)$, mesonbaryon $(\mathrm{mB})$ or meson-meson $(\mathrm{mm})$ collisions in the course of the heavy-ion collision. We consider the contri- 
butions from the photon decays of the following mesons:

$$
\begin{aligned}
\pi^{0} & \rightarrow \gamma+\gamma, \\
\eta & \rightarrow \gamma+\gamma, \\
\eta^{\prime} & \rightarrow \rho+\gamma, \\
\omega & \rightarrow \pi^{0}+\gamma, \\
\phi & \rightarrow \eta+\gamma, \\
a_{1} & \rightarrow \pi+\gamma . \\
\Delta & \rightarrow \gamma+N,
\end{aligned}
$$

The decay probability is calculated according to the corresponding branching ratios taken from the latest compilation by the Particle Data Group [63], updating slightly the values applied in earlier HSD investigations at SPS energies [41]. The broad resonances - including the $a_{1}, \rho, \omega$ mesons - in the initial or final state are treated in PHSD in line with their (in-medium) spectral functions as implemented and described in detail in Ref. [41].

The photon production from the mesonic decays represents a 'background' for the search of the direct photons. However, this background can only partly be fixed by the independent measurements. One usually uses the 'cocktail' method to estimate the photon decay spectra and their contribution to the elliptic flow $v_{2}$, which relies among others on the $m_{T}$-scaling assumption for the particle spectra. We have assumed throughout that the direct photon spectra do not include the contributions from the $\pi, \eta, \eta^{\prime}$ and $\omega$ decays, because they were subtracted experimentally.

\section{THEORETICAL DEVELOPMENTS}

The extensions compared to our previous publications on photon production in heavy-ion collisions within the PHSD model are described in the following subsection: an improved implementation of the Bremsstrahlung channel $m+m \rightarrow m+m+\gamma$ beyond the SPA is described in subsection III A an estimation of the suppression due to the Landau-Pomeranchuk-Migdal (LPM) effect will be presented in subsection III and additional baryonic processes will be described in subsection IIIC.

\section{A. Bremsstrahlung $m+m \rightarrow m+m+\gamma$ beyond the soft-photon approximation}

In the present work, we improve the description of the photon bremsstrahlung in meson+meson scattering by going beyond the soft-photon approximation [64]. Since pions are the dominant meson species in the heavy-ion collisions, we concentrate here on the description of the bremsstrahlung photon production in pion+pion collisions. Indeed, we recall that the pion+pion interactions are most numerous and provide the dominant source of photons from the meson+meson bremsstrahlung mechanism. This was shown in the previous investiga- tion [6] explicitly by studying the channel decomposition of the bremsstrahlung photons. Therefore, we will be able to considerably reduce the theoretical uncertainty in modeling the total photon production in the hadron bremsstrahlung by improving our modeling of the $\pi+\pi \rightarrow \pi+\pi+\gamma$ process. We will suggest a generalization to the other meson species and meson+baryon interactions in the end of this section.

In order to calculate the differential cross sections for the photon production in the processes of the type $\pi+\pi \rightarrow \pi+\pi+\gamma$, we use the one-boson exchange (OBE) model as originally applied in Ref. 32] to the dilepton bremsstrahlung in pion+pion collisions, later on in Ref. 65] to the low-energy photon bremsstrahlung in pion+pion and kaon+kaon collisions.

In order to achieve a reliable level of accuracy we model the interactions of pions with hadrons using a covariant microscopic effective theory with the interaction Lagrangian,

$$
L_{i n t}=g_{\sigma} \sigma \partial_{\mu} \vec{\pi} \partial^{\mu} \vec{\pi}+g_{\rho} \vec{\rho}^{\mu} \cdot\left(\vec{\pi} \times \partial_{\mu} \vec{\pi}\right)+g_{f} f_{\mu \nu} \partial^{\mu} \vec{\pi} \cdot \partial^{\nu} \vec{\pi},
$$

as suggested in Refs. 32, 61]. Within this model the interaction of pions is described by the exchange of scalar, vector and tensor resonances: $\sigma, \rho$ and $f_{2}(1270)$, respectively. Additionally, the form factors are incorporated in the vertices in the $t$ - and $u$-channels to account for the composite structure of the mesons and thus to effectively suppress the high momentum transfers:

$$
h_{\alpha}\left(k^{2}\right)=\frac{m_{\alpha}^{2}-m_{\pi}^{2}}{m_{\alpha}^{2}-k^{2}}
$$

where $m_{\alpha}=m_{\sigma}$ or $m_{\rho}$ or $m_{f}$ is the mass of the exchanged meson and $k^{2}$ is the momentum transfer squared. by

The cross section for $\pi+\pi \rightarrow \pi+\pi$ scattering is given

$$
\frac{d \sigma_{e l}(s)}{d t}=\frac{\left|M_{e l}\right|^{2}}{16 \pi s\left(s-4 m_{\pi}^{2}\right)},
$$

where the matrix element $|M|^{2}$ is calculated by coherently summing up the Born diagrams of the $\sigma-, \rho$ - and $f_{2}$-meson exchange in $t, s$ and $u$ channels (the $u$-channel diagrams are needed only in case of identical pions),

$$
\begin{aligned}
\left|M_{e l}\right|^{2}= & \mid M^{s}(\sigma)+M^{t}(\sigma)+M^{u}(\sigma) \\
& +M^{s}(\rho)+M^{t}(\rho)+M^{u}(\rho) \\
& +M^{s}(f)+M^{t}(f)+\left.M^{u}(f)\right|^{2} .
\end{aligned}
$$

Let us define the four-momenta of the incoming pions as $p_{a}=\left(E_{a}, \vec{p}_{a}\right)$ and $p_{b}=\left(E_{b}, \vec{p}_{b}\right)$, the momenta of the outgoing pions as $p_{1}=\left(E_{1}, \vec{p}_{1}\right)$ and $p_{2}=\left(E_{2}, \vec{p}_{2}\right)$ and the four-momentum of the exchanged resonance $(\sigma, \rho$ or $\left.f_{2}\right)$ as $k$.

The propagators of the massive and broad scalar and vector particles are used to describe the exchange of the $\sigma$ and $\rho$ mesons (see e.g. Ref. [32]). The resonance $f_{2}$ is a 


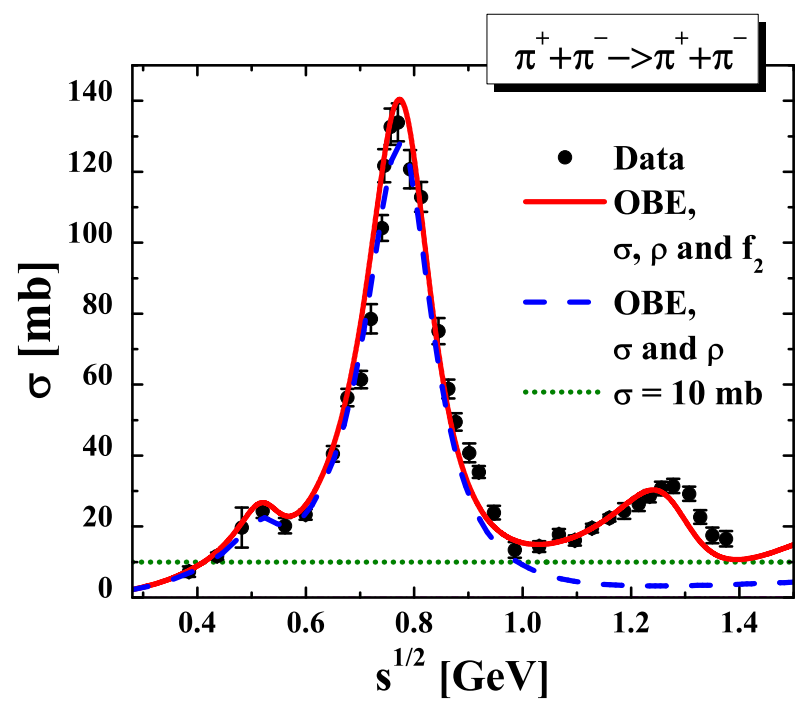

FIG. 1: (Color on-line) Cross section of pion+pion elastic scattering within two effective models are compared to the experimental data from [67, 68]: the exchange of two mesonic resonances, scalar $\sigma$ and vector $\rho$ (blue dashed line), and the exchange of three resonances $\sigma, \rho$ and the tensor resonance $f_{2}(1270)$ of the particle data booklet 63] (red solid line). The green dotted line shows the constant $\sigma_{e l}=10 \mathrm{mb}$ for comparison.

spin-2 particle, for which the full momentum-dependent propagator has been derived in [66]. The polarization sum is

$$
\begin{aligned}
P_{\mu \nu \alpha \beta} & =\frac{1}{2}\left(g_{\mu \alpha} g_{\nu \beta}+g_{\mu \beta} g_{\nu \alpha}-g_{\mu \nu} g_{\alpha \beta}\right) \\
& -\frac{1}{2}\left(g_{\mu \alpha} \frac{k_{\nu} k_{\beta}}{m_{f}^{2}}+g_{\mu \beta} \frac{k_{\nu} k_{\alpha}}{m_{f}^{2}}+g_{\nu \alpha} \frac{k_{\mu} k_{\beta}}{m_{f}^{2}}+g_{\nu \beta} \frac{k_{\mu} k_{\alpha}}{m_{f}^{2}}\right) \\
& +\frac{2}{3}\left(\frac{1}{2} g_{\mu \nu}+\frac{k_{\mu} k_{\nu}}{m_{f}^{2}}\right)\left(\frac{1}{2} g_{\alpha \beta}+\frac{k_{\alpha} k_{\beta}}{m_{f}^{2}}\right)
\end{aligned}
$$

Following the example of the dilepton production study in Ref. [32], we use the same propagator for the $f_{2}$ resonance while additionally accounting for its finite width by adding an imaginary part to the self-energy in accordance with the lifetime.

As the result, the following expressions are obtained for the matrix elements of the elastic $\pi+\pi$ scattering diagrams (we give here explicitly the $t$ - and $s$-channel results, the $u$-channels can be easily obtained by the cross- ing relations):

$$
\begin{aligned}
& M^{t}(\sigma)=\frac{-g_{\sigma}^{2} h_{\sigma}^{2}(t)\left(2 m_{\pi}^{2}-t\right)^{2}}{t-m_{\sigma}^{2}+i m_{\sigma} \Gamma_{\sigma}}, \\
& M^{s}(\sigma)=\frac{-g_{\sigma}^{2}\left(s-2 m_{\pi}^{2}\right)^{2}}{s-m_{\sigma}^{2}+i m_{\sigma} \Gamma_{\sigma}}, \\
& M^{t}(\rho)=\frac{-g_{\rho}^{2} h_{\rho}^{2}(t)(s-u)^{2}}{t-m_{\rho}^{2}+i m_{\rho} \Gamma_{\rho}}, \\
& M^{s}(\rho)=\frac{g_{\rho}^{2}(u-t)^{2}}{s-m_{\rho}^{2}+i m_{\rho} \Gamma_{\rho}}, \\
& M^{t}(f)=\frac{g_{f}^{2} h_{f}^{2}(t)}{t-m_{f}^{2}+i m_{f} \Gamma_{f}} \\
& \times \frac{1}{2}\left(\frac{2}{3}\left(2 m_{\pi}^{2}-t\right)^{2}-\left(s-2 m_{\pi}^{2}\right)^{2}-\left(2 m \pi^{2}-u\right)^{2}\right), \\
& M^{s}(f)=\frac{g_{f}^{2}}{s-m_{f}^{2}+i m_{f} \Gamma_{f}} \\
& \times \frac{1}{2}\left(\frac{2}{3}\left(s-2 m_{\pi}^{2}\right)^{2}-\left(2 m_{\pi}^{2}-t\right)^{2}-\left(2 m \pi^{2}-u\right)^{2}\right),
\end{aligned}
$$

where the Mandelstamm variables are defined as $s=$ $\left(p_{a}+p_{b}\right)^{2}=\left(p_{1}+p_{b}\right)^{2}, t=\left(p_{a}-p_{1}\right)^{2}=\left(p_{b}-p_{2}\right)^{2}$, $u=\left(p_{a}-p_{2}\right)^{2}=\left(p_{b}-p_{1}\right)^{2}$.

We point out that the formulae (11) are compact, because the masses of all pions were assumed to be equal to $m_{\pi}$ and the energy-momentum conservation $p_{a}+p_{b}=p_{1}+p_{2}$ was assumed. These conditions are not satisfied for the off-shell $\pi+\pi \rightarrow \pi+\pi$ subprocess, which we will encounter in the subsequent calculation of the bremsstrahlung photon production $\pi+\pi \rightarrow \pi+\pi+\gamma$. For the actual calculation we will use the off-shell generalizations $M\left(p_{a}, p_{b}, p_{1}, p_{2}\right)$ of the formulae (11), which we derive from the Feynman diagrams, but the off-shell formulae are too lengthy to be presented here.

A reduced version of the model with the exchange of only two resonances - the scalar $\sigma$ and the vector $\rho$ meson - was used by the authors of Ref. [65] to calculate the rate of the photon production from the $\pi+\pi \rightarrow \pi+\pi+\gamma$ process at low transverse momenta of the photons $\left(p_{T}<\right.$ $0.4 \mathrm{GeV})$. This approximation is suitable at low $p_{T}$ because the photon rate in this kinematical region is dominated by pion collisions of low center-of-mass energy $\sqrt{s}$, for which the contribution of the $f_{2}$-exchange is small. However, relatively high transverse momenta of photons $p_{T}=1-2 \mathrm{GeV}$ are of interest for our goal of clarifying the "puzzling" high elliptic flow of direct photons. Thus we need a robust model for the interaction of mesons also at $\sqrt{s}>1 \mathrm{GeV}$. Therefore, we use the OBE model with three mesons as interaction carriers (including the tensor particle $f_{2}(1270)$ ) in our present calculations.

Phenomenological coupling constants, masses and widths of the three interaction-carriers that enter the Lagrangian (6) have to be fixed to the integrated energydependent cross section of the pion+pion elastic scattering $\sigma_{e l}(s)$, which is known experimentally. We present 


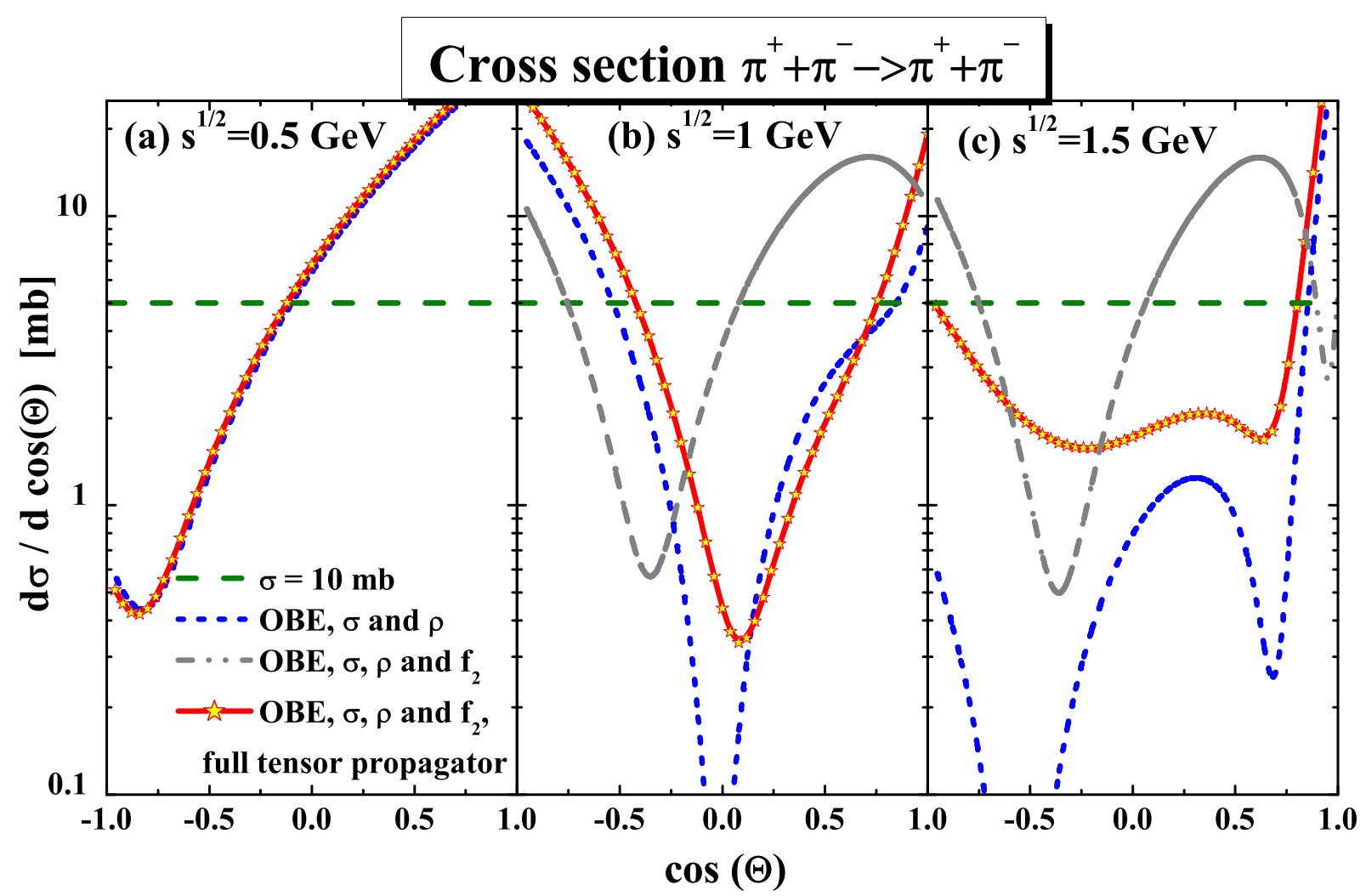

FIG. 2: (Color on-line) Angular differential cross section for pion+pion elastic scattering within two effective models: the exchange of two mesonic resonances, scalar $\sigma$ and vector $\rho$ (blue dashed line), and the exchange of three resonances $\sigma$, $\rho$ and the tensor particle $f_{2}(1270)$ (red solid line with symbols and gray dashed line). The red solid line with star symbols shows the model with the full momentum dependence of the $f_{2}$ propagator, while the grey dashed line is obtained neglecting the momentum dependence of the $f_{2}$ propagator. The green dashed line shows the constant and isotropic $\sigma_{e l}=10 \mathrm{mb}$ for comparison.

in Fig. 1 the integrated cross section of the $\pi+\pi$ elastic scattering in the two versions of the OBE model described above: taking into account 2 resonances $\sigma, \rho$ (dashed blue line) and taking into account 3 resonances $\sigma, \rho$ and $f_{2}$ (solid red line). Fitting the parameters of both variants of the OBE model (with two- or threeresonance exchange), the data from [67, 68] can be described. The best-fit parameters for the model used here are: $g_{\sigma} m_{\sigma}=2.0, m_{\sigma}=0.525 \mathrm{GeV}, \Gamma_{\sigma}=0.100 \mathrm{GeV}$, $g_{\rho}=6.15, m_{\rho}=0.775 \mathrm{GeV}, \Gamma_{\rho}=0.15 \mathrm{GeV}, g_{f} m_{f}=8.0$, $m_{f}=1.274 \mathrm{GeV}, \Gamma_{f}=0.18 \mathrm{GeV}$. The values of the masses and widths suggest the identification of the $\rho$ resonance to the $\rho$-meson and of the particle $f_{2}$ to the $f_{2}(1270)$ in the particle data book [63].

One sees in Fig. 1 that the tensor particle $f_{2}$ is important for the description of the pion interaction at higher collision energies $\sqrt{s}>1 \mathrm{GeV}$. Neglecting the contribution of the $f_{2}$ leads to an underestimation of the $\pi+\pi$ elastic scattering cross section by an order of magnitude around $\sqrt{s}=1.2-1.3 \mathrm{GeV}$. Later data on the $\pi+\pi$ interaction at $\sqrt{s}$ above $1 \mathrm{GeV}$ - extracted in Ref. [69] from the measurement of the $K+p \rightarrow \Lambda+\pi+\pi$ reaction - also point to the importance of the tensor interaction in the resonance region of the $f_{2}(1270)$.

With the parameters fixed to the integrated pion elastic scattering cross section as described above, we can calculate the differential cross section $d \sigma_{e l}(s) / d t$, which is a necessary ingredient for the calculation of the bremsstrahlung photon production within the softphoton approximation [61] for the low photon energy and low $\sqrt{s}$ (see below). The results of our calculations for the differential cross section of the process $\pi+\pi \rightarrow \pi+\pi$ as a function of the scattering angle $\Theta$ in the $\pi+\pi$ centerof-mass system is presented in Fig. 2 within two effective OBE models: including the exchange of two mesonic resonances, scalar $\sigma$ and vector $\rho$ (blue dashed line), and including the exchange of three resonances $\sigma, \rho$ and the tensor particle $f_{2}$ (red solid line with symbols and gray dashed lines). The red solid line with star symbols shows the latter model with the full momentum dependence of the $f_{2}$ propagator, while the grey dashed line is obtained, if the momentum dependence of the $f_{2}$ propagator is neglected. The green dashed line shows the constant and isotropic $\sigma_{e l}=10 \mathrm{mb}$ for comparison.

One can see the pronounced influence of the tensor resonance exchange at higher $\sqrt{s}$ of the collision. One ob- 

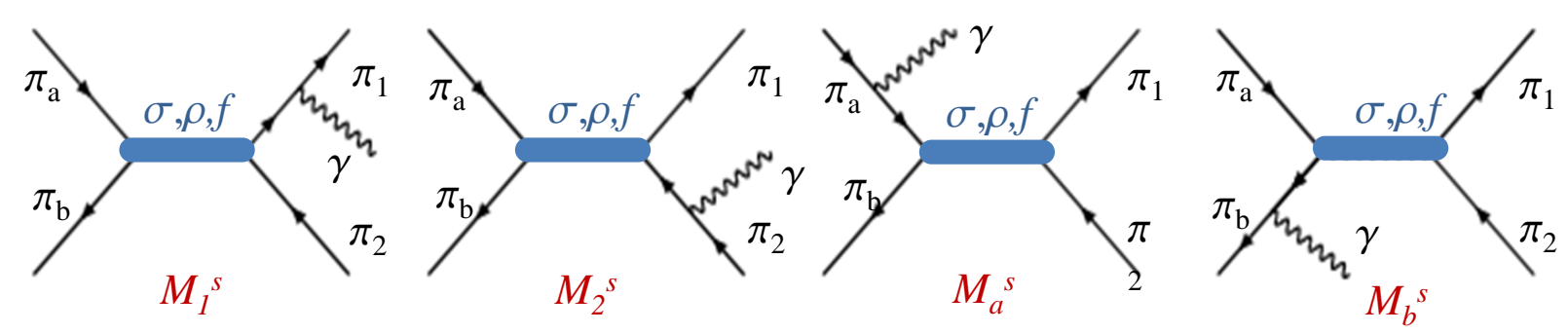

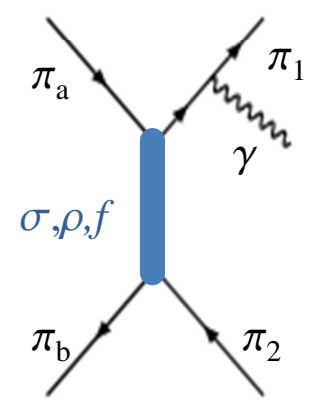

$M_{l}{ }^{t}$

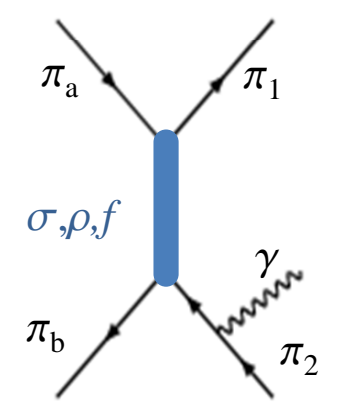

$M_{2}{ }^{t}$

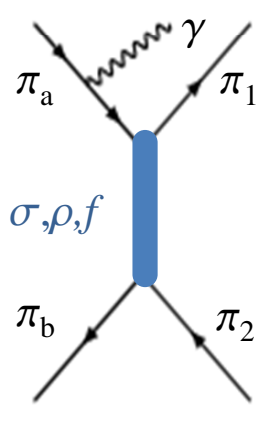

$M_{a}{ }^{t}$

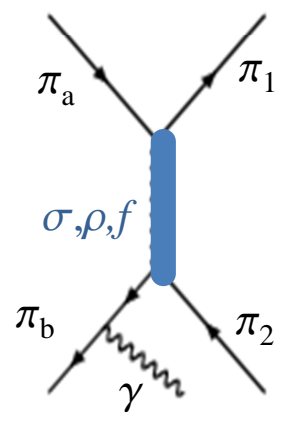

$M_{b}{ }^{t}$

FIG. 3: (Color on-line) Feynman diagrams for photon production in the reaction $\pi+\pi \rightarrow \pi+\pi+\gamma$ in the one-boson exchange (OBE) model. The time goes from left to right. For identical pions, e.g. $\pi^{+}+\pi^{+}$, the $u$-channel diagrams have to be added.

serves that for low $\sqrt{s}=0.5 \mathrm{GeV}$ the $\sigma$ peak dominates the cross section and the angular dependence is governed fully by the combination of scalar and vector terms. On the other hand, the influence of the $f_{2}$-resonance is clearly seen at $\sqrt{s}=1 \mathrm{GeV}$ and $\sqrt{s}=1.5 \mathrm{GeV}$ : the model with two resonances (dashed blue line) differs from the results of the other OBE variants. The correct momentum dependence of the $f_{2}$ propagator is important, as one sees from the difference of the star red and grey dashed lines.

Figs. 1 and 2 demonstrate that the inclusion of the tensor resonance in the model leads not only to an increase of the cross section for $\sqrt{s}>1 \mathrm{GeV}$ (in agreement with the data) but also to a considerable "flattening" of the $\cos \Theta$ distribution. The cross section at higher $\sqrt{s}$ in the extended model is more isotropic.

Using the OBE model (described above) for the covariant interactions of pions, we can calculate the emission of photons by the colliding pions by gauge coupling to the external hadron lines. The Feynman diagrams for the photon production in the process $\pi+\pi \rightarrow \pi+\pi+\gamma$ are shown in Fig. 3. For identical pions, e.g. $\pi^{+}+\pi^{+}$, the $u$-channel diagrams have to be added, which are obtained from the $t$-channel diagrams by exchanging the outgoing pions. The applicability of this method is not limited by the low energy of the photon but is restricted only by the applicability of the effective model to the description of the pion (elastic) interaction.

Let us again denote the four-momenta of the incoming pions by $p_{a}$ and $p_{b}$, the momenta of the outgoing pions by $p_{1}$ and $p_{2}$, while the photon momentum will be denoted by $q=\left(q_{0}, \vec{q}\right)$. The cross section for photon production in the process

$$
\pi\left(p_{a}\right)+\pi\left(p_{b}\right) \rightarrow \pi\left(p_{1}\right)+\pi\left(p_{2}\right)+\gamma(q)
$$

is given by

$$
d \sigma^{\gamma}=\frac{1}{2 \sqrt{s\left(s-4 m_{\pi}^{2}\right)}}|M(\gamma)|^{2} d R_{3},
$$

where $d R_{3}$ is the three-particle phase space, which depends on the momenta of the outgoing pions and of the photon,

$$
\begin{aligned}
d R_{3}= & \frac{d^{3} p_{1}}{(2 \pi)^{3} 2 E_{1}} \frac{d^{3} p_{2}}{(2 \pi)^{3} 2 E_{2}} \frac{d^{3} q}{(2 \pi)^{3} 2 q_{0}} \\
& (2 \pi)^{4} \delta^{4}\left(p_{a}+p_{b}-p_{1}-p_{2}-q\right) .
\end{aligned}
$$

The cross section (13) will be integrated over the final pion momenta to obtain $d \sigma / d^{3} q$. The $\delta$-function allows to perform four integrations analytically and the remaining two are done numerically.

The matrix element $M$ in (13) is a coherent sum of the diagrams presented in Fig. 3 - i.e. of the photon attached to each pion line $\pi_{a}, \pi_{b}, \pi_{1}$ and $\pi_{2}$ - and of contact terms, which account for the emission from the vertices and the internal lines:

$$
\begin{aligned}
|M(\gamma)|^{2} & =M_{\mu}^{*}(\gamma) M^{\mu}(\gamma) \\
& =\left|M_{a}^{\mu}+M_{b}^{\mu}+M_{1}^{\mu}+M_{2}^{\mu}+M_{c}^{\mu}\right|^{2} .
\end{aligned}
$$

The complex matrix elements for the photon emission from each of the pion lines $M_{i}^{\mu}$ are calculated as sums of 
the three meson exchanges $\left(\sigma, \rho, f_{2}\right)$. For instance:

$$
\begin{aligned}
M_{1}^{\mu}= & e J_{1}^{\mu}\left[M_{e l}^{s}\left(p_{a}, p_{b}, p_{1}+q, p_{2}\right)\right. \\
& +M_{e l}^{t}\left(p_{a}, p_{b}, p_{1}+q, p_{2}\right) \\
& \left.+M_{e l}^{u}\left(p_{a}, p_{b}, p_{1}+q, p_{2}\right)\right],
\end{aligned}
$$

with

$$
\begin{gathered}
J_{a, b}^{\mu}=-Q_{a, b} \frac{\left(2 p_{a, b}-q\right)^{\mu}}{2 p_{a, b} \cdot q}, \\
J_{1,2}^{\mu}=Q_{1,2} \frac{\left(2 p_{1,2}-q\right)^{\mu}}{2 p_{1,2} \cdot q},
\end{gathered}
$$

where $Q_{i}$ are the charges of the pions in terms of the electron charge $e$. The matrix elements for the pion elastic subprocess $M_{e l}\left(p_{a}, p_{b}, p_{1}+q, p_{2}\right)$ are the off-shell generalizations of the formulae (11).

The contact term $M_{c}^{\mu}$ is taken from Ref. [65], eq. (14), where it was derived by demanding the gauge invariance of the resulting cross section. Indeed, the gauge invariance of the result often has to be restored [70] in calculations within effective models. In the present work, we have used the contact terms in order to cancel the gaugedependent parts in the matrix element as in Ref. 65]. Alternatively, one can take into account additional diagrams with the emission of photons from the internal lines (see Refs [32]) but this method does not always eliminate the need for contact terms (see Ref. [70]). We have verified that $q_{\mu} M^{\mu}(\gamma)=0$. Comparing our results to calculations with a different gauge-fixing method will allow to quantify the uncertainty of the effective model applied (work in progress).

Within the soft-photon approximation (SPA) 62] one assumes that the strong interaction vertex is on-shell. The soft photon approximation is based on the first-order expansion in the Low theorem [64] and is valid at low photon energy and low $\sqrt{s_{m m}}$ of the meson+meson collision, as has been studied in detail for the production of dileptons in Ref. [32]. In this case the strong interaction part and the electromagnetic part can be separated, i.e. the soft-photon cross section for the reaction $m_{1}+m_{2} \rightarrow m_{1}+m_{2}+\gamma$ can be written as

$$
q_{0} \frac{d \sigma^{\gamma}(s)}{d^{3} q}=\frac{\alpha_{E M}}{4 \pi^{2}} \int_{-\lambda\left(s, m_{a}^{2}, m_{b}^{2}\right) / s}^{0}|\epsilon \cdot J(q, t)|^{2} \frac{d \sigma_{e l}(s)}{d t} d t,
$$

where $\alpha_{E M}$ is the fine structure constant, $t$ is the momentum transfer squared in the $\pi+\pi \rightarrow \pi+\pi$ subprocess, and $\epsilon$ is the photon polarization. $J^{\mu}$ is the electro-magnetic current

$$
J^{\mu}=-Q_{a} \frac{p_{a}^{\mu}}{\left(p_{a} \cdot q\right)}-Q_{b} \frac{p_{b}^{\mu}}{\left(p_{b} \cdot q\right)}+Q_{1} \frac{p_{1}^{\mu}}{\left(p_{1} \cdot q\right)}+Q_{2} \frac{p_{2}^{\mu}}{\left(p_{2} \cdot q\right)} .
$$

The polarization sum

$$
|\epsilon \cdot J|^{2}=\left\{\sum_{\text {pol } \lambda} J \cdot \epsilon_{\lambda} J \cdot \epsilon_{\lambda}\right\}
$$

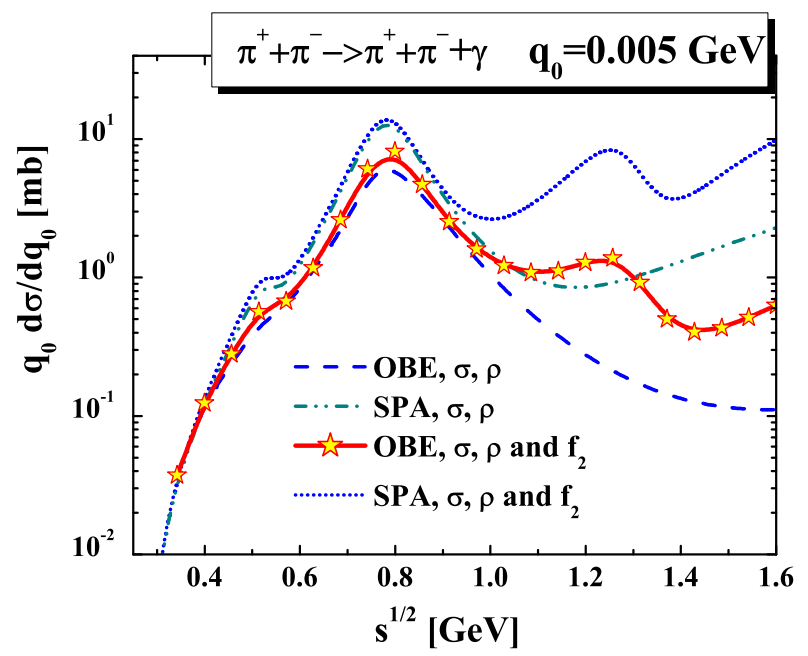

FIG. 4: (Color on-line) Cross section for the production of a photon with energy $q_{0}=0.005 \mathrm{GeV}$ in the process $\pi+\pi->$ $\pi+\pi+\gamma$ within the following models: the exact OBE cross section within the effective model taking into account scalar, vector and tensor interactions via the exchange of $\sigma, \rho$ and $f_{2}$ (1270)-mesons gives (red line with star symbols), the soft photon approximation to this model (blue dotted line); the OBE result within the model taking into account only the scalar and vector interactions via the exchange of $\sigma$ and $\rho$ mesons (blue dashed line), and the soft photon approximation to this model (cyan dash-dot-dotted line).

depends on the photon momentum $q$, the charges of the pions $Q_{i}$ as well as on the invariant kinematic variables, including $t$. For the case of equal-mass particle scattering $\left(m_{a}=m_{b}=m_{1}=m_{2}=m_{\pi}\right)$, one obtains [61]:

$$
\begin{aligned}
& |\epsilon \cdot J|^{2}=\frac{1}{q_{0}^{2}}\left\{-\left(Q_{a}^{2}+Q_{b}^{2}+Q_{1}^{2}+Q_{2}^{2}\right)\right. \\
& -2\left(Q_{a} Q_{b}+Q_{1} Q_{2}\right) \frac{s-2 m_{\pi}^{2}}{\sqrt{s\left(s-4 m_{\pi}^{2}\right)}} \ln \left(\frac{\sqrt{s}+\sqrt{s-4 m_{\pi}^{2}}}{\sqrt{s}-\sqrt{s-4 m_{\pi}^{2}}}\right) \\
& +2\left(Q_{a} Q_{1}+Q_{b} Q_{2}\right) \frac{2 m_{\pi}^{2}-t}{\sqrt{t\left(t-4 m_{\pi}^{2}\right)}} \ln \left(\frac{\sqrt{-t+4 m_{\pi}^{2}}+\sqrt{-t}}{\sqrt{-t+4 m_{\pi}^{2}}-\sqrt{-t}}\right) \\
& +2\left(Q_{a} Q_{2}+Q_{b} Q_{3}\right) \frac{s-2 m_{\pi}^{2}+t}{\sqrt{(s+t)\left(s+t-4 m_{\pi}^{2}\right)}} \\
& \left.\times \ln \left(\frac{\sqrt{s+t}+\sqrt{s+t-4 m_{\pi}^{2}}}{\sqrt{s+t}-\sqrt{s+t-4 m_{\pi}^{2}}}\right)\right\} \cdot(20)
\end{aligned}
$$

In (18), $d \sigma_{e l}(s) / d t$ is the on-shell differential elastic $\pi+\pi$ cross section, which is a function of the invariant energy $s$ and the pion scattering angle via $t$.

The expression (18) is considerably simpler in comparison to the "exact" OBE formula (13) because of the factorization of the diagrams from Fig. 3 into the electromagnetic part and the elastic $\pi+\pi \rightarrow \pi+\pi$ sub-process, for the cross section of which the $q$-dependence is omitted. This corresponds to neglecting the off-shellness of 
the pion, which emits the photon, e.g. for the pion $a$ :

$$
p_{a}-q \approx p_{a}
$$

Consequently, the sub-process invariant energy $s_{2}$ is also approximated by the total invariant energy of the process $\pi+\pi \rightarrow \pi+\pi+\gamma$ :

$$
s_{2} \equiv\left(p_{a}+p_{b}-q\right)^{2} \approx\left(p_{a}+p_{b}\right)^{2}=s,
$$

and the limits of integration over $t$ are also taken as for the on-shell case, i.e. from $-\lambda\left(s, m_{a}^{2}, m_{b}^{2}\right) / s$ to 0 , while the actual integration over the full 3-particle phase space in the exact treatment (13) involves different limits for $t$.

In Fig. 4 we show the resulting cross sections for the photon production in the process $\pi+\pi \rightarrow \pi+\pi+\gamma$ within the following models: the "exact" OBE taking into account scalar, vector and tensor interactions via the exchange of $\sigma, \rho$ and $f_{2}(1270)$-mesons gives the red line with star symbols, the soft photon approximation (18) to this model is shown by the blue dotted line; the OBE result within the model taking into account only the scalar and vector interactions via the exchange of $\sigma$ and $\rho$ mesons is shown by the blue dashed line, and the soft photon approximation to this model is presented by the cyan dash-dot-dotted line. The photon energy is fixed to $q_{0}=5 \mathrm{MeV}$. For the very low energy of the photon of $q_{0}=5 \mathrm{MeV}$ the SPA agrees with the "exact" cross section very well in the region of $\sqrt{s}<0.9 \mathrm{GeV}$ (see Fig. (4). However, the discrepancy to the OBE result is increasing rapidly with growing $\sqrt{s}$; the calculations for the higher photon energy of $q_{0}=0.5 \mathrm{GeV}$ show an even larger discrepancy between the SPA and the exact OBE result.

Using the cross section for the $\pi+\pi \rightarrow \pi+\pi+\gamma$ reaction according to (18) as a function of the photon energy $q_{0}$ and the collision energy $\sqrt{s}$, we calculate the yield $d N / d^{3} q$ and the invariant rate $q_{0} d R / d^{3} q$ of bremsstrahlung photon production from an equilibrated pion gas. Within kinetic theory, the rate of photon production in the collisions of particles $a$ and $b$ in a thermalized medium (number of photons produced per unit space-time volume $\left.d^{4} x\right)$ is an integral over the threemomenta of the incoming particles:

$$
\begin{aligned}
q_{0} \frac{d N}{d x^{4} d^{3} q}=g \int d s & \int \frac{d^{3} p_{a}}{(2 \pi)^{3}} \int \frac{d^{3} p_{b}}{(2 \pi)^{3}} e^{-\left(E_{a}+E_{b}\right) / T} \\
& \times v_{r e l} q_{0} \frac{d \sigma^{\gamma}}{d^{3} q} \delta\left(s-\left(p_{a}+p_{b}\right)^{2}\right),
\end{aligned}
$$

where $T$ is the temperature, $v_{r e l}$ is the relative velocity given by

$$
v_{r e l}=\frac{\sqrt{\left(p_{a} \cdot p_{b}\right)^{2}-m_{a}^{2} m_{b}^{2}}}{E_{a} E_{b}}
$$

and $g=\left(2 s_{a}+1\right)\left(2 s_{b}+1\right)$ is the spin degeneracy factor. Integrating the expression (23) over the particle momenta

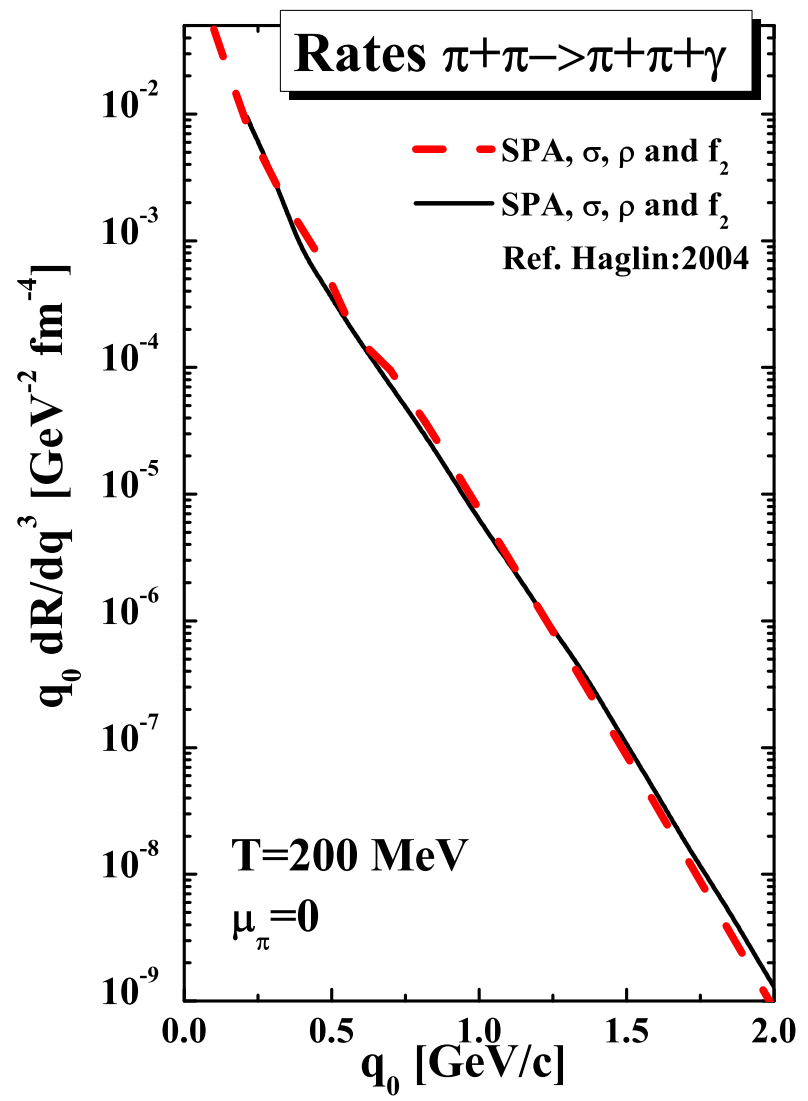

FIG. 5: (Color on-line) Invariant rate of the bremsstrahlung photon production from an equilibrated pion gas at a temperature of $T=200 \mathrm{MeV}$ and pion chemical potential $\mu_{\pi}=0$ as calculated in the OBE model with three resonance exchange within the soft-photon approximation (red dashed line). The black solid line "Haglin:2004" from Ref. [71] is shown for comparison.

one obtains [61]:

$$
q_{0} \frac{d N}{d^{4} x d^{3} q}=\frac{T^{6} g}{16 \pi^{4}} \int_{z_{\min }}^{\infty} d z \frac{\lambda\left(z^{2} T^{2}, m_{a}^{2}, m_{b}^{2}\right)}{T^{4}} K_{1}(z) q_{0} \frac{d \sigma^{\gamma}}{d^{3} q},
$$

where $z_{\min }=\left(m_{a}+m_{b}\right) / T, z=\sqrt{s} / T$, and $K_{1}(z)$ is the modified Bessel function.

The expression (23) can be generalized to account for quantum effects such as Bose enhancement or Pauli blocking (depending on the particle type) by integrating additionally over the momenta of the final particles and changing the Boltzmann distributions to the Fermi or Bose distribution functions $f_{i}(T)$ :

$$
\begin{array}{r}
q_{0} \frac{d N}{d x^{4} d^{3} q}=g \int d s \int \frac{d^{3} p_{a}}{(2 \pi)^{3}} \int \frac{d^{3} p_{b}}{(2 \pi)^{3}} \int \frac{d^{3} p_{1}}{(2 \pi)^{3}} \int \frac{d^{3} p_{1}}{(2 \pi)^{3}} \\
f_{a}(T) f_{b}(T)\left(1-f_{1}(T)\right)\left(1-f_{2}(T)\right) \\
\times v_{r e l} q_{0} \frac{d \sigma^{\gamma}}{d^{3} q} \delta\left(s-\left(p_{a}+p_{b}\right)^{2}\right) .
\end{array}
$$

In the current section we calculate the thermal rates ac- 
cording to formula (23). However, within the PHSD transport approach for the heavy-ion collisions in section IV the effects of the quantum statistics will be taken into account.

In Fig. 5 the rates are presented for a temperature $T=$ $200 \mathrm{MeV}$ and pion chemical potential $\mu_{\pi}=0$ for the OBE model with three resonance exchanges adopting the softphoton approximation (red dashed line). We confirm the results from Ref. [71] (black solid line) calculated within the same assumptions (SPA, three resonances) but with a slightly different parameter set of the Lagrangian. It is, however, questionable that the SPA is applicable at high photon energies.

We note that the accuracy of the SPA approximation can be significantly improved and the region of its applicability can be extended by slightly modifying the formula (18) - i.e. by evaluating the on-shell elastic cross section at the invariant energy $s_{2}$ of the sub-process. The latter is kinematically fixed to

$$
s_{2}=s-q_{0} \sqrt{s} \neq s,
$$

Thus the modified SPA formula is

$$
q_{0} \frac{d \sigma^{\gamma}(s)}{d^{3} q}=\frac{\alpha_{E M}}{4 \pi} \int_{-\lambda\left(s_{2}, m_{a}^{2}, m_{b}^{2}\right) / s_{2}}^{0}|\epsilon \cdot J(q, t)|^{2} \frac{d \sigma_{e l}\left(s_{2}\right)}{d t} d t .
$$

In the following, we will denote the approximation (28) as "improved SPA" and will show below that it provides a good description of the exact photon production rates.

But first we describe a simple model for the photon production in meson+meson collisions by the bremsstrahlung mechanism, i.e. the approximation of a constant meson + meson elastic cross section. We have seen above that the elastic-scattering cross section of pions is approaching about $\sigma=10 \mathrm{mb}$ at high $\sqrt{s}$ and becomes increasingly isotropic. As a very simple estimate for the interaction of two mesons one can use a constant isotropic elastic-scattering cross section of, e.g., $\sigma=10 \mathrm{mb}$ at all $\sqrt{\mathrm{s}}$. In case of the isotropic cross section $\sigma_{e l}$, it can be taken out of the integral (18). The integration of the electromagnetic current over the photon angle in (18) can be done in a straightforward way in case of small momentum transfer [61], since

$$
\int_{\text {tmin }}^{0} t d t=\frac{\left(s-4 m_{\pi}^{2}\right)^{2}}{2} .
$$

This leads to a useful approximation to the cross section for the photon bremsstrahlung in meson+meson collisions

$$
\begin{aligned}
q_{0} \frac{d^{3} \sigma^{\gamma}}{d^{3} q} & =\frac{\alpha}{4 \pi} \frac{\bar{\sigma}(s)}{q_{0}^{2}} \\
\bar{\sigma}(s) & =\frac{s-\left(M_{1}+M_{2}\right)^{2}}{2 M_{1}^{2}} \sigma(s),
\end{aligned}
$$

where $M_{1}$ and $M_{2}$ are the masses of the colliding mesons; here the first meson is assumed to have unit charge and the second meson to be charge-neutral, i.e. the approximation does not take into account the full dependence on the charges of the pions as was done in (20) or (17). This "constant-cross-section" approximation is useful in particular for an estimate of the photon bremsstrahlung in elastic collisions of mesons, for which the scattering cross sections are not known experimentally. We recall that the formulae (30) and (31) have been used to model photon bremsstrahlung in the collisions of various meson species in the previous transport calculations in Refs. [6, 7, 41].

Finally, we proceed to calculate the photon production rates beyond the soft photon approximation, using the cross section for the $\pi+\pi \rightarrow \pi+\pi+\gamma$ reaction calculated according to the exact OBE expression (13). We present the calculated invariant rate $q_{0} d R / d q^{3}$ of bremsstrahlung photons produced from an equilibrated pion gas at $T=$ $150 \mathrm{MeV}$ and $\mu_{\pi}=40 \mathrm{MeV}$ in Fig. 6. The results of the following models are compared:

- model 1 (red solid line): exact rates within the one-boson exchange model (OBE) beyond the softphoton approximation - i.e. using the formula (13) for the photon production cross section $q_{0} d \sigma^{\gamma} / d^{3} q$;

- model 2 (blue dotted line): result within the soft photon approximation - i.e. using the formula (18) - while using the elastic $\pi+\pi$ cross section calculated within the OBE model as given by the equations (8)-(11);

- model 3 (black short-dashed line): results of the improved soft photon approximation - i.e. using the formula (28) instead of (18) - and the same pion elastic scattering cross section as in the model 2 ;

- model 4 (green dashed line): soft photon approximation using a constant isotropic elastic cross section of $\sigma_{e l}=10 \mathrm{mb}$ and assuming for the pion charges $Q_{a}=Q_{1}=1, Q_{b}=Q_{2}=0$-i.e. using the formula (30). For this case the elastic cross section does not depend on $\sqrt{s}$ and therefore there is no difference between the SPA and improved SPA.

The rate of bremsstrahlung photons at low transverse momenta $p_{T}<0.4 \mathrm{GeV}$ has been calculated before in Ref. 65] within the one-boson exchange model with the exchange of two resonances for the same system. This previous result is shown for comparison by the cyan dashed line and is confirmed by our present calculations. The agreement is expected, since our calculations differ only in the inclusion of the $f_{2}$-meson exchange, which is important for large $\sqrt{s}$ and does not play an important role for the production of low transverse momentum photons, which is dominated by low $\sqrt{s}$ of the $\pi+\pi$ collisions.

On the other hand, the SPA (model 2) deviates from the exact OBE result (model 1) even at low $q_{0}$, because the former directly follows the $\sqrt{s}$ structure of the elastic $\pi \pi$ cross section. Since the formula (18) does not account 


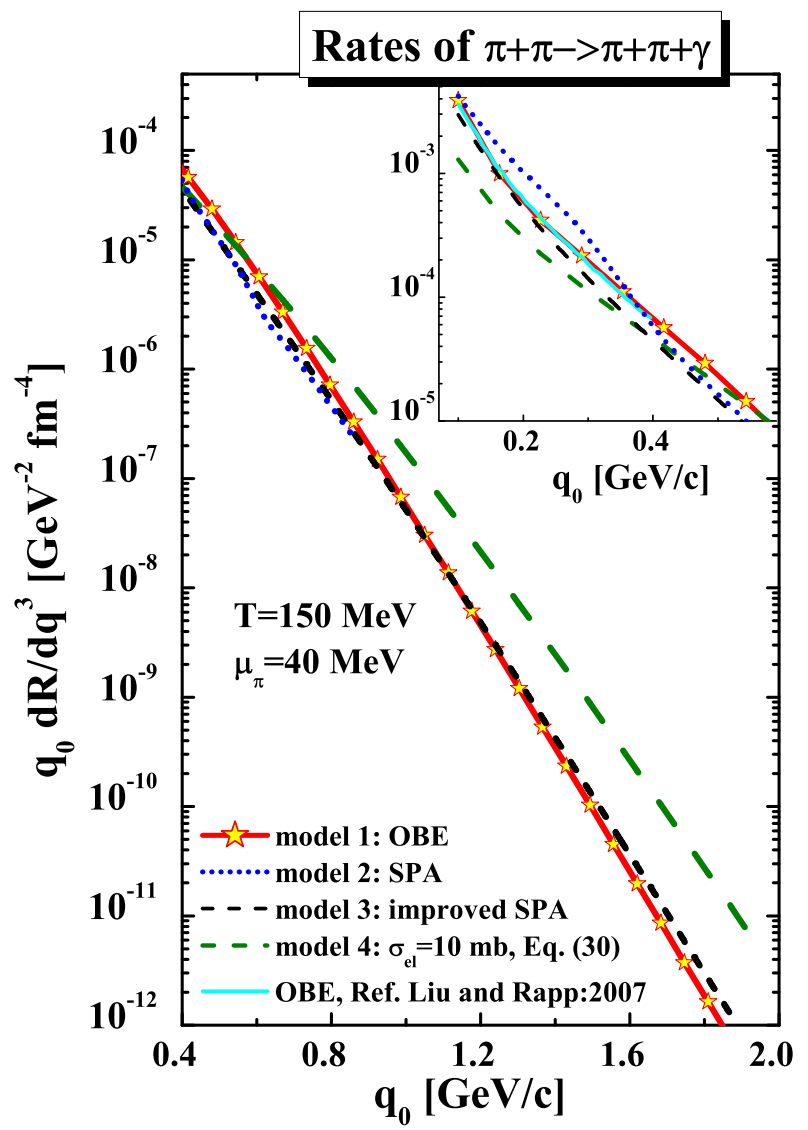

FIG. 6: (Color on-line) Invariant rate of bremsstrahlung photons produced from an equilibrated pion gas at $T=150 \mathrm{MeV}$ and $\mu_{\pi}=40 \mathrm{MeV}$ versus the photon energy $q_{0}$. The inlet shows the same quantity for the range of photon energies $q_{0}=0.1-0.4 \mathrm{GeV}$. The calculations have been performed within the following models: (1) OBE model beyond the softphoton approximation (red solid line with star symbols); (2) OBE model within the soft photon approximation (blue dotted line); (3) OBE model within the improved soft photon approximation (black short-dashed line) - the invariant energy $s_{2}$ of the on-shell $\pi+\pi$ elastic process is not equal to the total invariant energy of the process $s: s_{2}=s-q_{0} \sqrt{s}$; (4) the soft photon approximation with the constant isotropic elastic cross section of $\sigma_{e l}=10 \mathrm{using}$ the formula (30) (green dashed line). The cyan solid line "Liu and Rapp:2007" from Ref. 65] is shown for comparison.

for the off-shellness of the emitting pion, it overweights the high- $\sqrt{s}$ part of the elastic cross section, in line with the findings of Refs. [32, 61]. We note that the OBE model presented here is constrained by the pion scattering data only up to $\sqrt{s_{\pi \pi}}=1.4 \mathrm{GeV}$ and generally cannot be extended to large $\sqrt{s}$. Thus the SPA scenario "model 2" is not reliable for large $q_{0}$ (approximately for $\left.q_{0}>0.8 \mathrm{GeV}\right)$. This is not the case for the improved SPA (model 3).

One can see in Fig. 6] that the improved SPA (28) gives a very good approximation to the exact result at higher photon energies of up to $q_{0} \approx 2 \mathrm{GeV}$. This is because the $\sqrt{s_{2}}$ of the sup-process does not reach such high values as $\sqrt{s}$, and the OBE model for the elastic cross section is sufficiently realistic in this region of $\sqrt{s_{2}}$.

In comparison, the constant cross-section approximation (based on formula (30) ) overestimates the exact rates for $q_{0}>1 \mathrm{GeV}$ and underestimates for $q_{0}<0.4 \mathrm{GeV}$. This model approximately corresponds to the procedure used previously in our transport calculations for the estimation of the photon bremsstrahlung in meson + meson collisions in Refs. 6, 7, 41]. In the present work, we will use the exact OBE cross section $d \sigma^{\gamma} / d^{3} q$. Therefore, we will now find a lower yield of bremsstrahlung photons for $q_{0}>1 \mathrm{GeV}$ in the transport simulations of heavy-ion collisions, see section IV below.

Another reasons for the good agreement between the "improved" SPA rates with the exact ones is that the dominant contribution to the rates comes from the low collision energies $\sqrt{s}$, while the deviation between the exact cross section of the process $\pi+\pi \rightarrow \pi+\pi+\gamma$ from that calculated within the improved SPA is most pronounced at high $\sqrt{s}$ and high $q_{0}$. Such high collision energies $\sqrt{s}$ are suppressed in a thermal medium exponentially by the Boltzman factor describing the occupation of the pion energies at fixed temperature. It is not clear, whether this high accuracy of the "improved SPA" holds also out-ofequilibrium. We show in Fig. 7 explicitly the distributions in the number of meson+meson, meson+baryon and parton+parton collisions versus their invariant energy $\sqrt{s}$ as calculated within the PHSD for the case of a $A u+A u$ collision with the energy $\sqrt{s_{N N}}=200 \mathrm{GeV}$ at impact parameter $b=7 \mathrm{fm}$, which is the centrality qualitatively similar to minimum bias. The distribution of meson+meson, meson+baryon and parton+parton collision invariant energies $d N / d \sqrt{s}$ in an actual heavy-ion reaction shows a clear dominance of the low $-\sqrt{s}$ comover collisions. Therefore we expect that the deviations of the cross section $\pi+\pi \rightarrow \pi+\pi+\gamma$ in the improved SPA from the exact ones at high $\sqrt{s}$ will not influence much the yield of the photons that result from the integration over all pion collisions:

$$
q_{0} \frac{d^{3} \sigma^{\gamma}(A A)}{d q^{3}}=\int d s \frac{d N_{\pi \pi}^{\text {coll }}}{d s} q_{0} \frac{d^{3} \sigma^{\gamma}(\pi \pi)}{d q^{3}} .
$$

In summarizing the results of the current section, we have improved the implementation of the photon bremsstrahlung in the process $\pi+\pi \rightarrow \pi+\pi+\gamma$ within the PHSD transport approach, using now the exact OBE cross sections beyond the soft-photon approximation. The bremsstrahlung photon production in collisions of other meson types is treated only approximately, i.e. in analogy to the $\pi+\pi$ collisions by means of mass-scaled cross sections.

We note, that another important source of the photons is the bremsstrahlung in meson+baryon collisions (cf. Ref. 7]). As we have shown above, the SPA gives a good approximation to the exact rates, if we use the correct invariant energy in the hadronic sub-process $s_{2}=s-q_{0} \sqrt{s}$ and a realistic model for the differential 


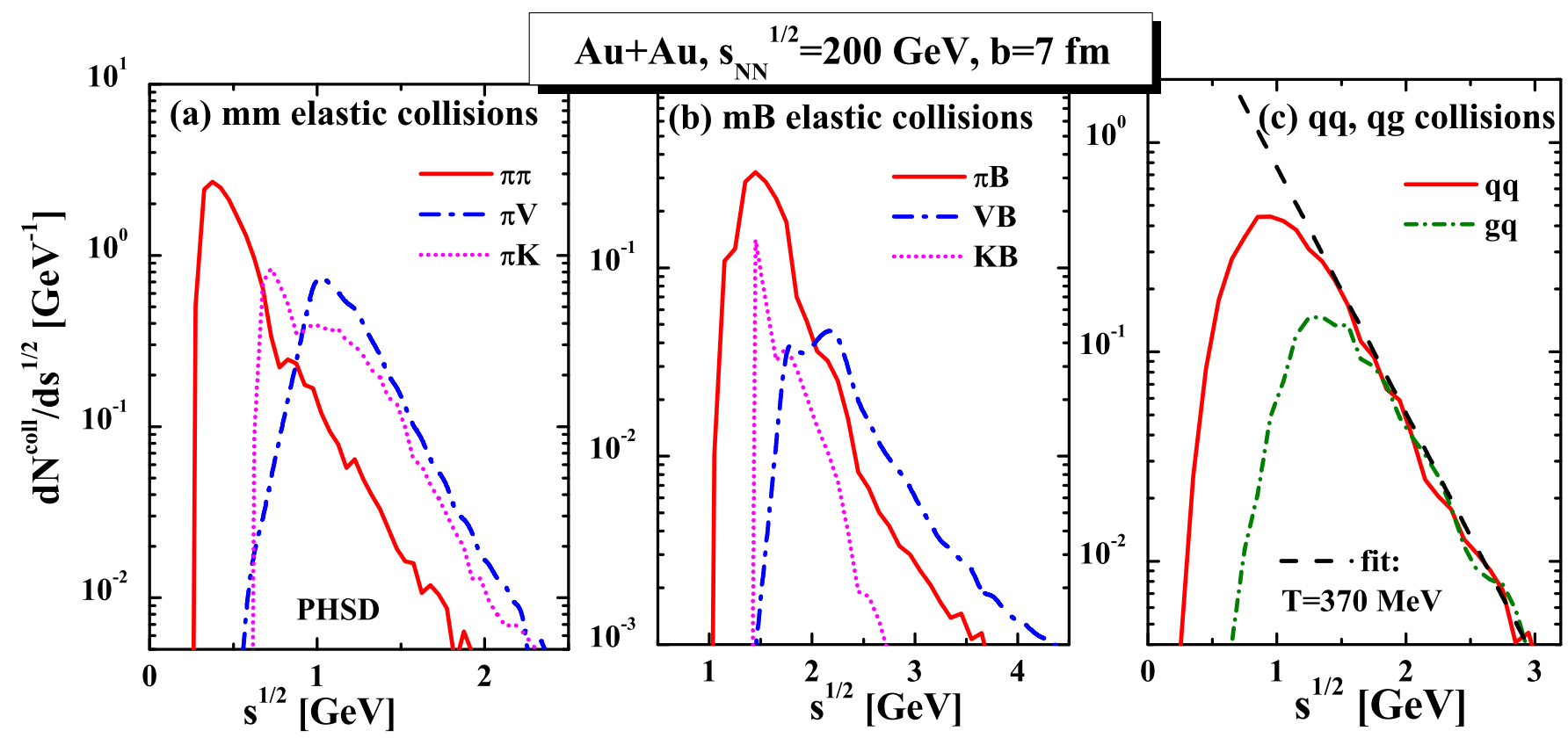

FIG. 7: (Color on-line) The distribution in the invariant collision energy $\sqrt{s}$ for the elastic scattering of mesons on mesons (left panel), mesons on baryons (middle panel) and for the elastic scattering of partons (right panel) in the course of a $A u+A u$ collision at $\sqrt{s_{N N}}=200 \mathrm{GeV}$ for $b=7 \mathrm{fm}$ as calculated within the PHSD approach. Here $V \equiv(\rho, \omega, \phi) ; K$ denotes all the strange mesons $K \equiv\left(K, \bar{K}, K^{*}, \bar{K}^{*}\right)$ and $B$ stands for the baryons $B=(p, n, \Delta, \ldots)$.

cross section of the subprocess, i.e. for the elastic scattering of mesons on baryons. The cross sections for the meson+baryon elastic scatterings (implemented within the PHSD transport approach) have been previously adjusted to the data differentially in energy and angular distribution. Thus we evaluate the photon production in the processes $m+B \rightarrow m+B+\gamma$ in the PHSD by using realistic elastic scattering cross sections taken at the correct invariant energy $\sqrt{s_{2}}$ in the scope of the improved SPA.

\section{B. The LPM effect}

The radiation of photons by charged particles is modified in the medium compared to the vacuum. One of such medium effects is caused by the absence of well-defined incoming and outgoing asymptotic states due to the multiple scattering of particles in a strongly interacting environment. If the subsequent scatterings occur within the time necessary for photon radiation $\tau_{\gamma} \sim 1 / q_{0}$, then the amplitudes for the emission of photons before and after the charged particle scattering have to be summed coherently. The effect of this destructive interference on the photon spectrum by electrons transversing a dense medium was first studied by Landau and Pomeranchuk in Ref. [72] and Migdal in Ref. 73]. Accordingly, the Landau-Pomeranchuk-Migdal (LPM) effect modifies the spectrum of photons produced in the medium in com- parison to the incoherent sum of emissions in quasi-free scatterings, leading especially to a suppression of the low energy photons because the formation time of the photon $\tau_{\gamma}$ is proportional to the inverse photon energy $1 / q_{0}$. In particular, the LPM effect regularizes the $1 / q_{0}$ divergence of the quasi-free bremsstrahlung spectra. The LPM suppression and the induced thermal mass of the medium quanta (the dielectric effect) together ensure that the photon spectrum is finite in the limit $q_{0} \rightarrow 0$.

The importance of the LPM effect for the case of dilepton and photon production from QCD systems was shown in Refs. 74 76]. The magnitude of the LPM suppression is governed by the average time between the collisions $\tau$, which in turn is given by the inverse scattering length $a$ or by the inverse average spectral width of the particles $\Gamma$ :

$$
\tau=\frac{1}{a} \approx \frac{1}{\Gamma} .
$$

The LPM suppression is more pronounced in case of small $\tau$, i.e. for high reaction rates. Thus we expect it to be important for the emission of photons from the strongly-interacting quark-gluon plasma (sQGP) as created in the early phase of the heavy-ion collision. Indeed, it was shown in Refs. [35, 77] in the scope of the DQPM that the average collision time of partons is as short as $\tau \approx 2-3 \mathrm{fm} / \mathrm{c}$ for temperatures in the range $T=1-2 T_{c}$, where $T_{c} \approx 158 \mathrm{MeV}$ is the deconfinement transition temperature. In comparison, the average time 


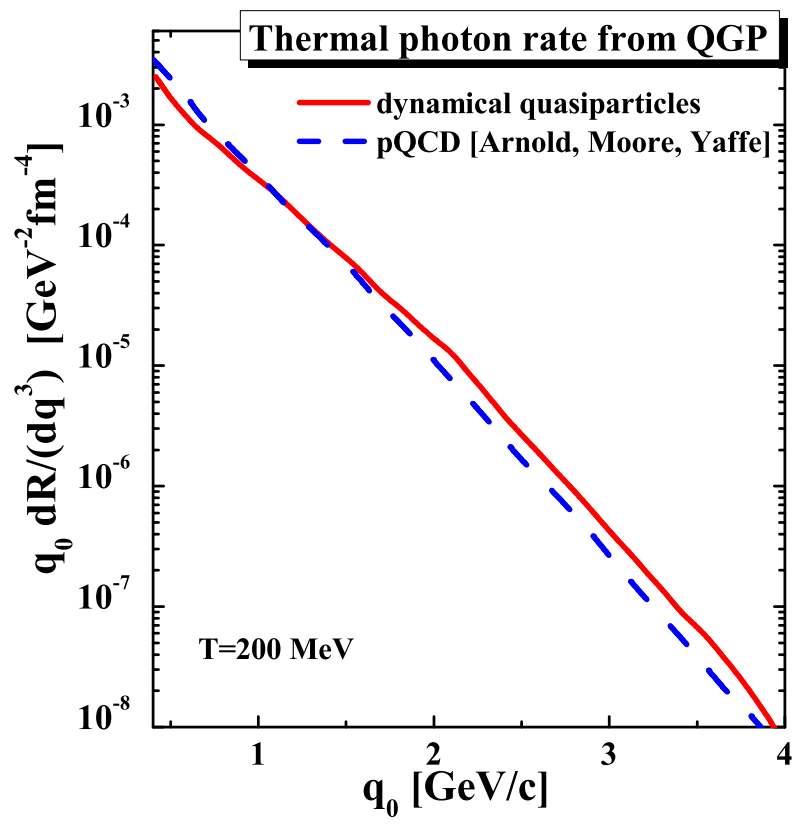

FIG. 8: (Color on-line) Invariant rate of photons produced from the strongly-interacting quark-gluon plasma consisting of massive broad quasi-particle quarks and gluons (red solid line). The leading-order pQCD rate (blue dashed line) from Ref. [78] is shown for comparison.

between pion collisions in a thermalized pion gas at temperatures $T<T_{c}$ is above $10 \mathrm{fm} / \mathrm{c}$, see Ref. [74].

The photon production in the sQGP proceeds by the interactions of dressed quarks and gluons through quark annihilation and gluon Compton scattering processes $q+\bar{q} \rightarrow g+\gamma, q(\bar{q})+g \rightarrow q(\bar{q})+\gamma$ as well as through the quark bremsstrahlung and off-shell parton decays. In the strongly interacting QGP (and in PHSD), the gluon and quark propagators differ significantly from the noninteracting propagators such that bare production amplitudes can no longer be used [37, 38]. The off-shell quarks and gluons have finite masses and widths, which parametrize a resummed interaction of the QGP constituents. The quark off-shellness leads to higher twist corrections $\left(\sim m_{q}^{2} / s, m_{q}^{2} / t, m_{q}^{2} / u\right)$ to photon and dilepton production cross sections [54, 79]. These corrections are small in hard hadron scattering at high center-ofmass energy $\sqrt{s}>10 \mathrm{GeV}$, but become substantial for photon production in the sQGP, where the characteristic $\sqrt{s}$ of parton collisions is of the order of a $\mathrm{GeV}$ (cf. r.h.s. of Fig. 7). Using the cross sections for photon radiation by dressed quarks and gluons in the processes $q \bar{q} \rightarrow g \gamma$ and $q g \rightarrow q \gamma$ as derived in Ref. [54], we have calculated the rates of photons produced in a thermalized strongly interacting QGP. Fig. 8 presents the invariant rate of photons produced from the strongly-interacting quark-gluon plasma at the temperature $T=200 \mathrm{MeV}$ (red solid line). The leading-order Log-resummed perturbative QCD rate (blue solid line) from Arnold, Moore and Yaffe (AMY rate) is taken from Ref. 78] and shown for comparison. One observes a qualitative agreement between the results of both models although the degreesof-freedom and their couplings are different. We mention that photon rates recently calculated at the NLO in perturbative QCD [80 82] also approximately are in line with those presented in Fig. 8

Let us now quantify the magnitude of the LPM effect on the spectrum of photons radiated from the QGP as calculated within the PHSD. The coherent photon production rate - taking into account the LPM effect - differs from the non-coherent cross section by a suppression factor, which generally depends on the photon energy, temperature and the interaction strength of the constituents. The coherent photon emission rate was derived in Ref. [4] for an elastically interacting pion gas in the soft photon approximation for the photon radiation amplitudes. The authors of Ref. 74] used the same method for the calculation of the photon emission over the whole trajectory of the charged particle as was adopted in the original work by Migdal in Ref. [73]. After averaging over the times between collisions $\xi$, assuming an exponential distribution

$$
\frac{d W}{d \xi}=a e^{-\xi a}
$$

the coherent photon emission rate was found to be

$$
\frac{d R}{d q^{3}}=N \frac{2 \alpha_{E M}}{(2 \pi)^{2}}\left\langle v^{2} \frac{\left(1-\cos ^{2} \Theta\right)}{a^{2}+q_{0}^{2}(1-v \cos \Theta)^{2}}\right\rangle,
$$

where the brackets $\langle$. $>$ stand for an average over the velocities after the scattering $(v, \cos \Theta)$, while $N$ is the number of scatterings and $\alpha_{E M} \approx 1 / 137$. A realistic parametrization of the data was used for the pion elastic scattering cross section (cf. section IIIA), but the scattering was assumed isotropic. The incoherent rate is obtained from (35) in the limit $a=0$.

An analytical form of the coherence factor was obtained in Ref. [76] in the model of hard scattering centers, using a quantum mechanical approach to coherently sum the photon amplitudes from all the scatterings. In the thermal medium the spatial distribution of the scattering centers is assumed random. Consequently, the function (34) naturally arises in this model for the distribution of times between collisions by a direct calculation of the two-particle correlation function. The quenching factor in the dipole limit $(\vec{q}=0)$ was found as

$$
\left(G\left(q_{0} \tau\right)\right)^{2}=\left(\frac{\left(q_{0} \tau\right)^{2}}{1+\left(q_{0} \tau\right)^{2}}\right)^{2}
$$

Although formula (36) was obtained in a simple model, it is useful because it correctly captures the dependence of the LPM suppression on the average strength of the interaction given solely by the mean-free-time between collisions $\tau$ in the assumption of isotropic collisions.

The perturbative interaction of quarks and gluons is dominated by small scattering angles due to the massless 
particle exchange in the $t$-channel diagrams. In this case the coherence factor for the quark system in the limit of small scattering angles was obtained in Ref. 75]. However, up to now the LPM effect in case of a strongly interacting QGP with dressed broad quasi-particles has not been evaluated. The elastic scattering of dressed quarks in the PHSD is not dominated by the $t \rightarrow 0$ pole as in the perturbative case since the gluon mass (of order $1 \mathrm{GeV}$ ) acts as a regulator in the amplitude. Accordingly, the angular distribution for quark-quark scattering is closer to an isotropic distribution for low or moderate $\sqrt{s}$ in accordance with the model assumptions of Ref. [76] such that the expression (36) should apply as an estimate of the LPM suppression for the photon emission within the PHSD.

In Fig. 9 we show the photon emission rate in a QGP at the temperature $T=190 \mathrm{MeV}$ as calculated in the PHSD as an incoherent sum of the photon emission in quark and gluon scatterings (red solid line). The blue dashed line gives the same rate with the quenching factor (36) applied using $\tau(T)=1 / \Gamma(T) \approx 3.3 \mathrm{fm} / \mathrm{c}$ from the DQPM (for $T=190 \mathrm{MeV}$ ). We observe that the suppression in comparison to the incoherent rate is visible only for photon energies $q_{0}<0.4 \mathrm{GeV}$. For an estimate of the upper limit on the LPM suppression we employ the relaxation time approximation for the ratio of the shear viscosity over entropy density $\eta / s$ which gives $\eta / s \approx 0.14$ at $T=190 \mathrm{MeV}$ in DQPM [23, 24]. The lowest bound as conjectured within the AdS/CFT correspondence is $\eta / s=1 /(4 \pi) \approx 0.08$. In the relaxation time approximation this corresponds to a lower value of $\tau \approx 1.9 \mathrm{fm} / \mathrm{c}$. The coherent photon rate in this case is given by the magenta dotted line and shows a peak in the photon rate for $q_{0} \approx 0.2 \mathrm{GeV}$.

In order to further clarify the strength of the LPM suppression of the photon emission in the sQGP, we use the knowledge of the electric conductivity $\sigma_{0}(T)$ of the sQGP from the DQPM [77] which is roughly in line with more recent results from lattice QCD. For details of the latter analysis on the electric and magnetic response of the sQGP we refer the reader to Ref. [83]. We recall that the photon emission rate from a thermal medium is controlled by $\sigma_{0}$ [84] via the relation,

$$
\frac{\sigma_{0}}{T}=\frac{8 \pi^{3}}{3 T^{2}} \lim _{q_{0} \rightarrow 0}\left(q_{0} \frac{d R}{d^{3} q}\right),
$$

where $T$ is the temperature of the system, $q_{0}$ is the photon energy and $\vec{q}$ is the photon momentum. Using the number for $\sigma_{0} / T$ from the PHSD at the temperature of $T=190 \mathrm{MeV}$ from Ref. 77, we obtain a limiting value for the scaled photon emission rate of 0.04 for $q_{0} \rightarrow 0$ according to formula (37) (green short dashed line in Fig. 9). The blue dashed line in Fig. 9 - the estimate of the rate based on formula (36) and the DQPM average spectral width of the quarks/antiquarks - indeed approaches the limiting value of 0.04 as given by the kinetic calculations of the electric conductivity in Refs. [77, 83].

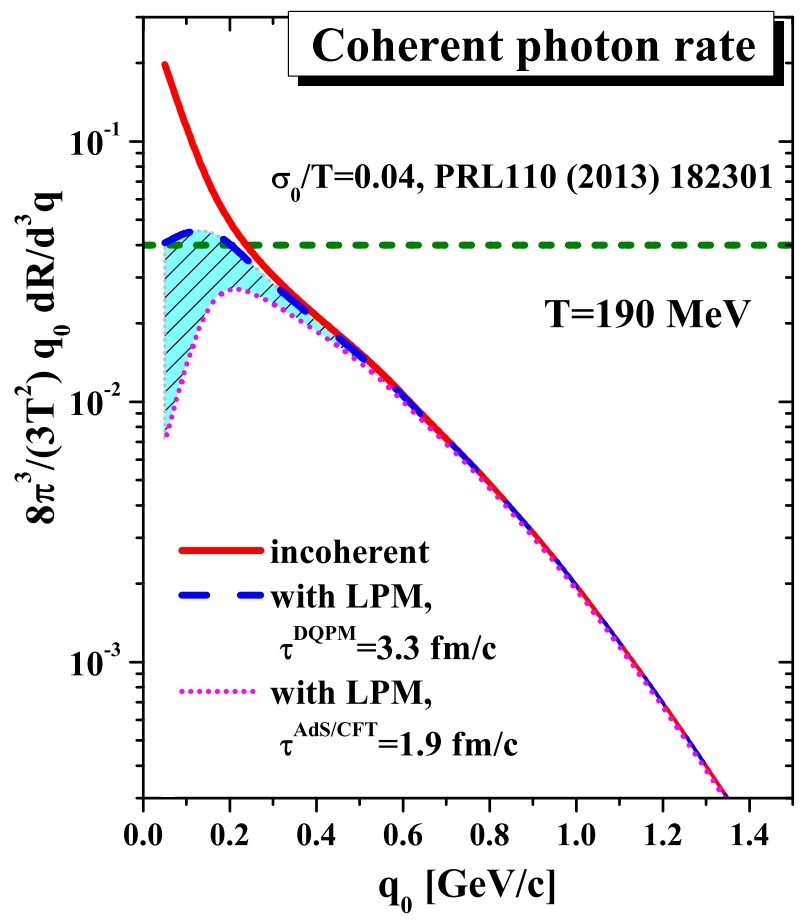

FIG. 9: (Color on-line) Incoherent invariant photon production rate from the strongly-interacting quark-gluon plasma consisting of massive broad quasi-particle quarks and gluons (red solid line) scaled by $8 \pi^{3} /\left(3 T^{2}\right)$ in order to match the electric conductivity for $q_{0} \rightarrow 0$ (cf. Eq. (37)). The blue dashed line and the magenta dotted line show the coherent rates with the two assumptions for the average time between the collisions $\tau$ - from the DQPM model and from the AdS/CFT correspondence.

Taking into account some uncertainty in the determination of $\tau$ and the expression (36), we conclude from Fig. 9 and analogous calculations at different temperatures that the LPM effect influences the photon production from the QGP at photon energies below $q_{0} \approx$ $0.4 \mathrm{GeV}$, but is negligible for higher photon energies. We note in passing that the suppression of the photon spectrum in the hadronic phase is much smaller due to the lower interaction rate, i.e. longer interaction time $\tau$.

\section{Additional meson-nucleon processes}

In the present work we incorporate into the PHSD approach additionally the $2 \rightarrow 2$ processes $V+N \rightarrow N+\gamma$, where $V$ stands for a vector meson while $N$ denotes a proton or neutron. These processes are the baryonic counterparts to the mesonic $2 \rightarrow 2$ reactions $\pi+\rho / \pi \rightarrow \gamma+\pi / \rho$. We here consider the interaction of nucleons with the mesons $V=\rho, \phi, \omega$, taking into account the various possible charge combinations, e.g. $\rho^{0}+p \rightarrow \gamma+p$, $\rho^{-}+p \rightarrow \gamma+n, \rho^{+}+n \rightarrow \gamma+p$, etc. Additionally, we take into account the photon production in the decay of the $\Delta$-resonance, $\Delta \rightarrow N+\gamma$. 
In order to evaluate the probabilities for photon production in the collisions of vector mesons with nucleons, we use the inverse processes $\gamma+N \rightarrow \rho+N$, $\gamma+N \rightarrow \phi+N, \gamma+N \rightarrow \omega+N$ (controlled by data) and employ detailed balance to obtain the differential cross sections for the processes $\rho+N \rightarrow \gamma+N, \phi+N \rightarrow \gamma+N$, $\omega+N \rightarrow \gamma+N$.

We recall that the detailed balance formula reads:

$$
\sigma(N V \rightarrow \gamma N)=\frac{g_{\gamma}}{g_{V}} \frac{p_{N \gamma}^{* 2}}{p_{N V}^{* 2}} \sigma(\gamma N \rightarrow N V),
$$

where $g_{\gamma}=2$ and $g_{V}=3$ are the spin degeneracy factors of the photon and the vector meson $V, p_{N \gamma}^{*}$ is the centerof-mass momentum in the $N+\gamma$ system, and $p_{N V}^{*}$ is the center-of-mass momentum in the $N+V$ system.

The cross sections for the exclusive photo-production of $\rho, \phi$ and $\omega$ vector mesons on the nucleon have been measured by the Aachen-Berlin-Bonn-HamburgHeidelberg-Munich (ABBHHM) Collaboration and published in Ref. [85]. In the same work also parametrizations for these cross section have been given that are based on the vector-meson-dominance model with a nonrelativistic Breit-Wigner (BW) spectral function for the $\rho$-meson. Later, the fits have been updated in Ref. [86] using relativistic BW spectral functions for $\rho, \omega$ and $\phi$ mesons.

The total cross sections - fitted in Ref. [86] to the data from Ref. [85] - are given by

$$
\sigma(\gamma N \rightarrow V N)=\frac{1}{p_{N \gamma}^{*} s} \int d \mu\left|M_{V}\right|^{2} p_{N V}^{*} A_{V}(\mu),
$$

where the mass of the vector meson is distributed by the spectral function $A_{V}(\mu)$ :

$$
A_{V}(\mu)=\frac{2}{\pi} \frac{\mu^{2} \Gamma(\mu)}{\left(\mu^{2}-M_{i}^{2}\right)^{2}+\mu^{2} \Gamma^{2}(\mu)},
$$

with $M_{i}$ denoting the pole mass of the meson. The matrix elements for $\gamma+N \rightarrow V+N$ are parametrized as

$$
\begin{aligned}
& \left|M_{\rho}\right|^{2}=0.16 \mathrm{mb} \mathrm{\textrm {GeV } ^ { 2 }} \text {, } \\
& \left|M_{\omega}\right|^{2}=\frac{0.08 p_{V N}^{* 2}}{2(\sqrt{s}-1.73 \mathrm{GeV})^{2}+p_{V N}^{* 2}} \mathrm{mb} \mathrm{GeV}^{2} \text {, } \\
& \left|M_{\phi}\right|^{2}=0.004 \mathrm{mb} \mathrm{GeV} \text {. }
\end{aligned}
$$

The cross sections (39) with the parameters (41) are consistent with the dynamics of vector mesons in the PHSD, where also relativistic BW spectral functions for vector mesons are used and propagated off-shell.

For the angular distribution of the $\rho$-meson production in the process $\gamma+N \rightarrow N+\rho$, we follow the suggestion of Ref. [86],

$$
\frac{d \sigma}{d t} \sim \exp (B t)
$$

with the photon-energy dependent parameter $B$ (fitted to the data): $B=5.7$ for $q_{0} \leq 1.8 \mathrm{GeV}, B=5.43$ for $1.8<$ $q_{0} \leq 2.5 \mathrm{GeV}, B=6.92$ for $2.5<q_{0} \leq 3.5 \mathrm{GeV}, B=8.1$ for $3.5<q_{0} \leq 4.5 \mathrm{GeV}, B=7.9$ for $q_{0}>4.5 \mathrm{GeV}$.

The data in Ref. 86] have shown that the cross section is dominated by the $t \approx 0$ region in line with the physics assumptions of the vector dominance model (VDM) where the process $\gamma+N \rightarrow V+N$ is described by the incident photon coupling to the vector meson of helicity \pm 1 , which consequently is scattered elastically by the nucleon (cf. Refs. 87 89]).

Let us now briefly describe the modeling of the photon production in the decays of the $\Delta$-resonance. The $\Delta \rightarrow N \gamma$ width depends on the resonance mass $M_{\Delta}$, which is distributed according to the $\Delta$ spectral function. Starting from the pioneering work of Jones and Scadron [90], a series of models 91 93] provided the mass-dependent electromagnetic decay width of the $\Delta$ in relation to the total width of the baryon. We employ the model of Ref. [92] in the present calculations where the spectral function of the $\Delta$ resonance is assumed to be of relativistic Breit-Wigner form. We adopt the "Moniz" parametrization [94] for the shape of the $\Delta$ spectral function, i.e. the dependence of the width on the mass $\Gamma^{\text {tot }}\left(M_{\Delta}\right)$.

\section{COMPARISON TO DATA FROM HEAVY-ION COLLISIONS}

Before presenting the results on photon spectra from heavy-ion collisions we stress again that the PHSD approach so far has provided a consistent description of the bulk properties of heavy-ion collisions - rapidity spectra, transverse mass distributions, azimuthal asymmetries of various particle species - from low SPS up to LHC energies [34, 35, 47]. Furthermore, dilepton production from hadronic and partonic sources has been calculated at SPS, RHIC and LHC energies [51 53] and successfully compared to the available dilepton data 95]. Accordingly, the global dynamics of the bulk matter, its collective flow as well as the electromagnetic emissivity in heavy-ion collisions appear to be well under control. Here we extend our previous photon studies in Refs. [6, 7] at the top RHIC energy also to the top SPS and LHC energy incorporating the improved cross sections as described above.

The inclusive photon yield as produced in $p+p$ and $A+A$ collisions is divided into "decay photons" and "direct photons". Decay photons - which constitute the major part of the inclusive photons - stem from the photonic decays of hadrons (mesons and baryons). These decays occur mainly at later times and outside of the active reaction region and therefore carry limited information on the initial high-energy state. Consequently, it is attempted to separate the decay photons from the inclusive yield (preferably by experimental methods) and to study the remaining, direct photons. One usually uses the "cocktail" method to estimate the contribution of the photon decays to the spectra and to the elliptic flow 
$v_{2}$, which relies among others on the $m_{T}$-scaling assumption and on the photon emission only by the finally produced hadrons with the (momenta) distributions as in the final state. Depending on the particular experimental set-up, different definitions of the decay photons are applied: whether only the decays of $\pi^{0}$ - and $\eta$-mesons are attributed to the decays photons or also the decays of the less abundant and shorter-living particles $\eta^{\prime}, \omega, \phi$, $a_{1}$ and the $\Delta$-resonance. Indeed, the determination of the latter contributions (in particular, $a_{1}, \Delta$ ) by experimental methods is questionable, because of the emission during the absorption and regeneration in the initial interacting phase. Therefore, the theoretical understanding of the decay photon contributions to the inclusive spectrum is important. Especially when analyzing simultaneously various measurements at different energies and within different experimental settings. One can consider theoretically decays photons from the following processes:

$$
\begin{gathered}
\pi^{0} \rightarrow \gamma+\gamma, \quad \eta \rightarrow \gamma+\gamma, \quad \eta^{\prime} \rightarrow \rho+\gamma, \\
\omega \rightarrow \pi^{0}+\gamma, \quad \phi \rightarrow \eta+\gamma, \quad a_{1} \rightarrow \pi+\gamma, \quad \Delta \rightarrow \gamma+N
\end{gathered}
$$

where the parent hadrons are produced in baryon-baryon $(B B)$, meson-baryon $(\mathrm{mB})$ or meson-meson $(\mathrm{mm})$ collisions in the course of the heavy-ion collision.

The following contributions to the direct photons have been identified so far:

- The photons at large transverse momentum $p_{T}$, so called "prompt" or "pQCD" photons, are produced in the initial hard $N+N$ collisions and the from the jet fragmentation, which are well described the perturbative QCD (pQCD) calculations. The latter, however, might be modified in $A+A$ contrary to $p+p$ due to the modification of the parton distributions (initial state effect) the parton energy loss in the medium (final state effect). In the $A+A$ collisions at large $p_{T}$, there may also be contributions from the induced jet- $\gamma$-conversion in the QGP and the jet-medium photons from the scattering of hard partons with thermalized partons $q_{\text {hard }}+q(g)_{Q G P} \rightarrow \gamma+q(g)$, but these are relatively small. The prompt photons are well modeled by using the perturbative calculations and adjusting them to the high- $p_{T}$ region of the observed direct photon yield.

- After the deduction of the prompt photons from the direct photon spectra, there is a significant remaining photon radiation at $p_{T}<3 \mathrm{GeV}$ observed, which is dubbed as "thermal" photons. The low- $p_{T}$ photons are emitted by the various partonic and hadronic sources as listed below:

1. Photons are radiated by quarks in the interaction with other quarks and gluons, such as in the reactions

$$
q+\bar{q} \rightarrow g+\gamma, \quad q / \bar{q}+g \rightarrow q / \bar{q}+\gamma
$$

In addition, photon production in the bremsstrahlung reactions $q+q / g \rightarrow q+q / g+\gamma$ are possible.

2. All colliding hadronic charges (meson, baryons) can also radiate photons by the bremsstrahlung processes:

$m+m \rightarrow m+m+\gamma \quad m+B \rightarrow m+B+\gamma$.

3. Additionally, the photons can be produced in binary meson+meson and meson+baryon collisions. We consider within the PHSD the direct photon production in the following $2 \rightarrow 2$ scattering processes:

$$
\begin{gathered}
\pi+\pi \rightarrow \rho+\gamma, \quad \pi+\rho \rightarrow \pi+\gamma \\
V+N \rightarrow \gamma+N
\end{gathered}
$$

accounting for all possible charge combinations.

\section{A. Direct photon spectra from SPS to LHC}

We start with the system $\mathrm{Pb}+\mathrm{Pb}$ at $\sqrt{s_{N N}}=17.3$ $\mathrm{GeV}$, i.e. at the top SPS energy. Fig. 10 shows the comparison of the PHSD calculations to the data of the WA98 Collaboration from 96] for 10\% centrality in the pseudorapidity interval $2.35<\eta<2.95$. In addition to the sources, which have been incorporated in the original HSD study [41], the meson+baryon bremsstrahlung, $V N \rightarrow N \gamma, \Delta \rightarrow N \gamma$ decay and the QGP channels are added. Compared to the earlier results of Ref. [41], the description of the data is further improved and the conclusions remain unchanged: the bremsstrahlung contributions are essential for describing the data at low $p_{T}$. This interpretation is shared by the authors of Refs. [65, 71, 97], who also stressed the importance of the meson+meson bremsstrahlung in view of the WA98 data using hydrodynamical or fireball models. Note that the photon contribution from the QGP is practically negligible for low $p_{T}$ and reaches at most $25 \%$ at $p_{T}>0.5 \mathrm{GeV}$.

We now step on to the top RHIC energy of $\sqrt{s_{N N}}$ $=200 \mathrm{GeV}$ and update our results on the differential photon spectra for the system $\mathrm{Au}+\mathrm{Au}$. The results for the direct photon spectrum as a sum of partonic as well as hadronic sources for the photons produced in minimum bias $\mathrm{Au}+\mathrm{Au}$ collisions is presented in Fig. 11 as a function of the transverse momentum $p_{T}$ at mid-rapidity $|y|<0.5$. This observable has been calculated within the PHSD earlier [6, 7], but has to be revisited in the present work since we have incorporated additional photon production channels such as the binary baryon + meson collisions $V+$ $N \rightarrow N+\gamma, \Delta \rightarrow N \gamma$ and improved the calculation of the $m+m \rightarrow m+m+\gamma$ bremsstrahlung channel based on the OBE model results which go beyond the soft-photon approximation. Indeed, a direct comparison 


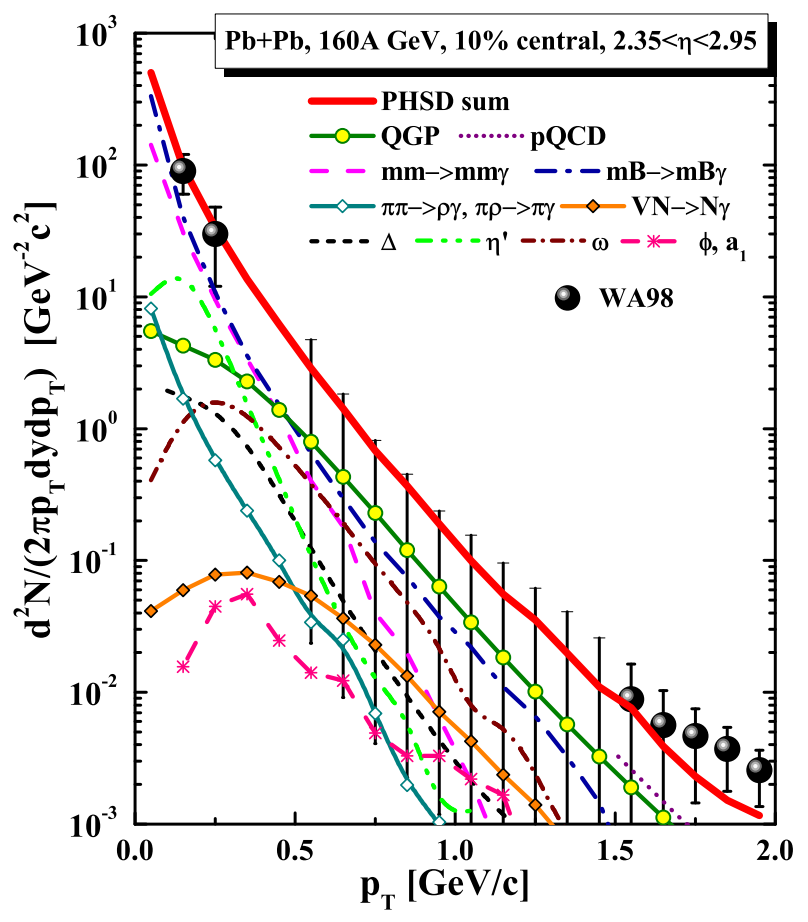

FIG. 10: (Color on-line) Comparison of the PHSD calculations to the data of the WA98 Collaboration from 96]. In comparison to the original HSD study [41]: (i) the meson+baryon bremsstrahlung (blue dash-dotted line), $\Delta$ decays (black short-dashed line) and the photons from QGP (green line with round symbols) are added (ii) and the meson + meson bremsstrahlung is now calculated beyond the SPA (magenta dashed line). The black line with diamond symbols labeled as "other" includes: $\omega, \eta^{\prime}, \phi$ an $\mathrm{d} a_{1}$-meson decays, binary channels $\pi+\rho / \pi \rightarrow \pi / \rho+\gamma$ and $N+V \rightarrow N+\gamma$.

of Fig. 11 to our previous results for the direct photon spectra at RHIC (cf. Fig. 1 in Ref. [7] and Fig. 3 of Ref. 6] ) shows that the data seem to favor the OBE model employed here in comparison to the SPA with the simple constant isotropic cross section employed earlier. In particular the agreement with the data is improved in the high- $p_{T}$ range.

We recall that in our calculations the direct photon spectrum has the following contributions: photon bremsstrahlung in meson+meson $m+m \rightarrow m+m+\gamma$ (blue dash-dotted line) and meson+baryon collisions $m+B \rightarrow m+B+\gamma$ (magenta dashed line); photon production in the QGP in the processes $q+\bar{q} \rightarrow g+\gamma$, and $q(\bar{q})+g \rightarrow q(\bar{q})+\gamma$ (green line with yellow circles); the reactions $\pi+\rho \rightarrow \pi+\gamma$ and $\pi+\pi \rightarrow \rho+\gamma$, (cyan line with open symbols); the meson+baryon reactions $V+N \rightarrow N+\gamma$ (orange line with filled symbols); decays of $\phi$ and $a_{1}$ mesons and of $\Delta$-baryons (black short-dashed line); the photon production in the initial hard collisions ("pQCD") is given by the hard photon yield in $\mathrm{p}+\mathrm{p}$ collisions scaled with the number of collisions $N_{\text {coll }}$ (violet dotted line). The measured transverse momentum spectrum $d N / d p_{T}$ (given by the filled circles) is reproduced

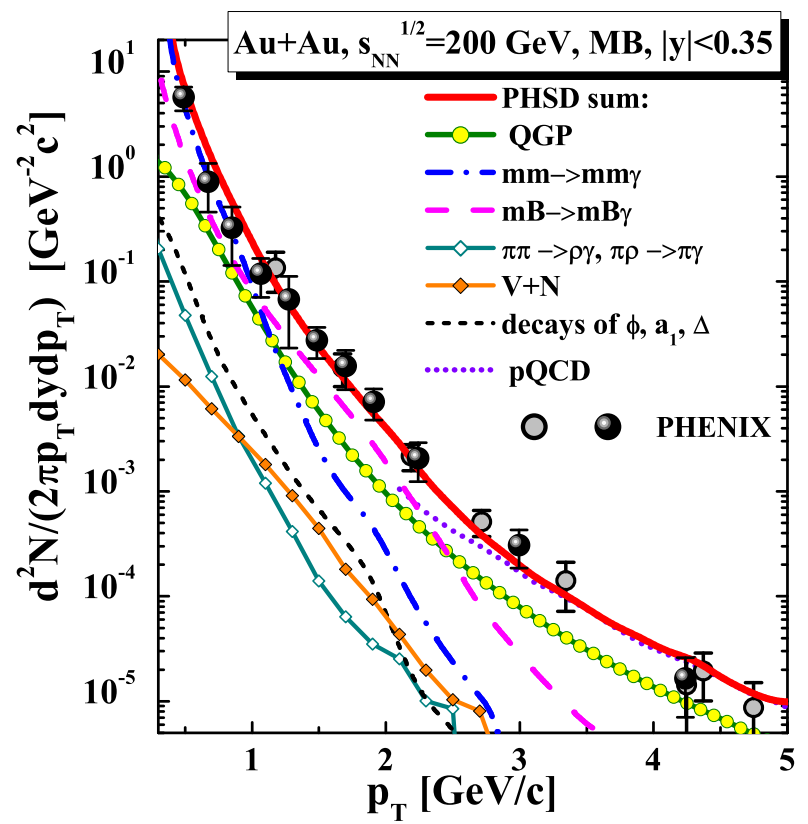

FIG. 11: (Color on-line) PHSD results for the spectrum of direct photons produced in minimum bias $\mathrm{Au}+\mathrm{Au}$ collisions at $\sqrt{s_{N N}}=200 \mathrm{GeV}$ as a function of the transverse momentum $p_{T}$ at mid-rapidity $|y|<0.5$. The data of the PHENIX Collaboration are taken from Refs. [30, 31]. For the individual lines see the legend in the figure.

well by the sum of all partonic and hadronic sources (red solid line).

As we have previously stressed in Ref. [7], the centrality dependence of the photon spectra carries additional information, which can be used to disentangle the hadronic and partonic sources of the photon production. The recent measurements by the PHENIX Collaboration [31] confirm the predictions within the PHSD from Ref. [7]. The centrality dependence of the integrated thermal photon yield in PHSD was found to scale as $N_{\text {part }}^{\alpha}$ with the exponent $\alpha \approx 1.5$, which is in a good agreement with the most recent measurement of $\alpha=1.48 \pm 0.08 \pm 0.04$ by the PHENIX Collaboration [31]. We compare our calculations and the data in Fig. 12, In the present work we have added the cross sections of the processes $V+N \rightarrow \gamma+N$ by exploiting detailed balance from the cross sections (39) and the photons from the $\Delta$-Dalitz decays. The contributions of these channels to the direct photon yield in the $A u+A u$ collisions at $\sqrt{s_{N N}}=200 \mathrm{GeV}$ at four different centralities are explicitly shown in Fig. 12, where the recent measurements by the PHENIX Collaboration 31] are compared to the PHSD spectra, too. It can be seen in Fig. 12 that the inclusion of the photon production from the $V+N$ reactions does not enhance the total direct photon yield much at all four centralities. We find that partonic channels and bremsstrahlung photon production "overshine" the $2 \rightarrow 2$ reactions, both meson+meson and (vector) meson+baryon channels. 

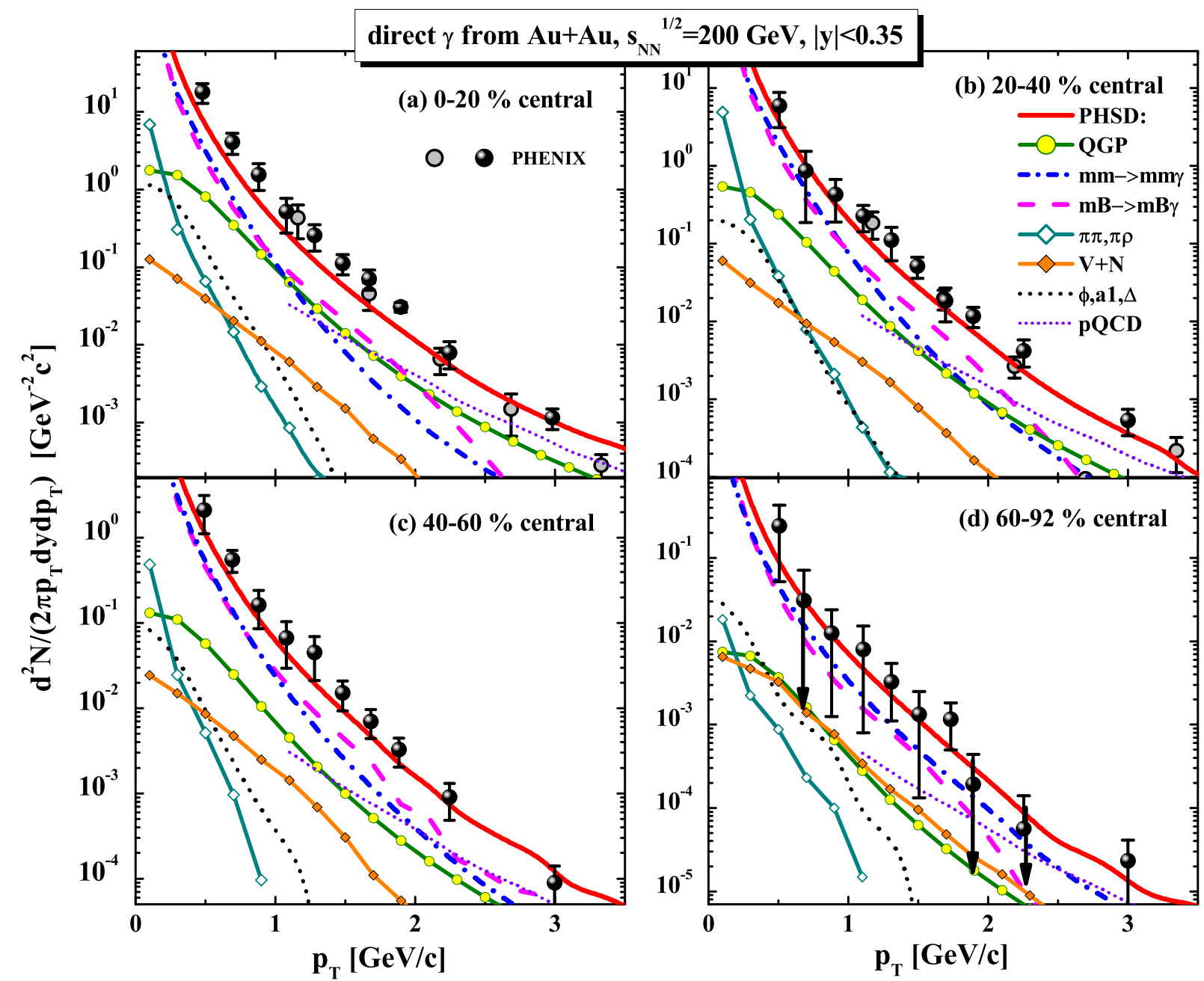

FIG. 12: (Color on-line) Contribution of the photon production in the two-to-two $\rho+$ nucleon interaction (orange dashed lines) to the total direct photon spectra (red lines) at the top RHIC energy for different centralities. The dominant sources are the photons from the QGP and from the hadronic two-to-three bremsstrahlung processes. The PHENIX data are from Refs. 30, 31].

The modification of vector meson spectral functions in the nuclear medium is an interesting phenomenon, which has been found essential for the understanding of dilepton production at SPS and RHIC energies [51, 52]. Accordingly, there should be also an effect of the in-medium modification of vector-meson spectral functions on the photon production in the $V+N$ reactions. However, since the $V+N$ channel does not dominate the spectrum at any photon energy, we conclude that this effect cannot be resolved in the total direct photon spectra at the present level of the data accuracy at RHIC; this has been explicitly shown earlier in Ref. [41] for the top SPS energy.

Next, let us investigate the photon production across the phase transition in the heavy-ion collision to check whether the observed yield of direct photons is produced dominantly in some particular region of the energydensity or in some particular phase of matter. Fig. 13 shows the yield of photons produced at mid-rapidity in 0$20 \%$ most central $A u+A u$ collisions at $\sqrt{s_{N N}}=200 \mathrm{GeV}$ as functions of the approximate local "temperature" (i.e. the fourth-root of the energy density) from the PHSD. The top panel of Fig. 13 presents the calculations for photons with the transverse momentum $p_{T}=0.5 \mathrm{GeV}$, while the bottom panel corresponds to photons with a transverse momentum $p_{T}=1.5 \mathrm{GeV}$. We observe that the early, hot state does not dominate the photon production in the QGP contrary to expectations of the static thermal fireball model, where photon production is roughly proportional to a power of the temperature $\left(\sim T^{4}\right)$. The integration over the dynamical evolution of the heavyion collision leads to roughly the same contribution of the different energy density regions since the rate decreases but the space-time volume increases. The photon production in the hadronic phase is dominated by the lower energies/temperatures because of the very long 

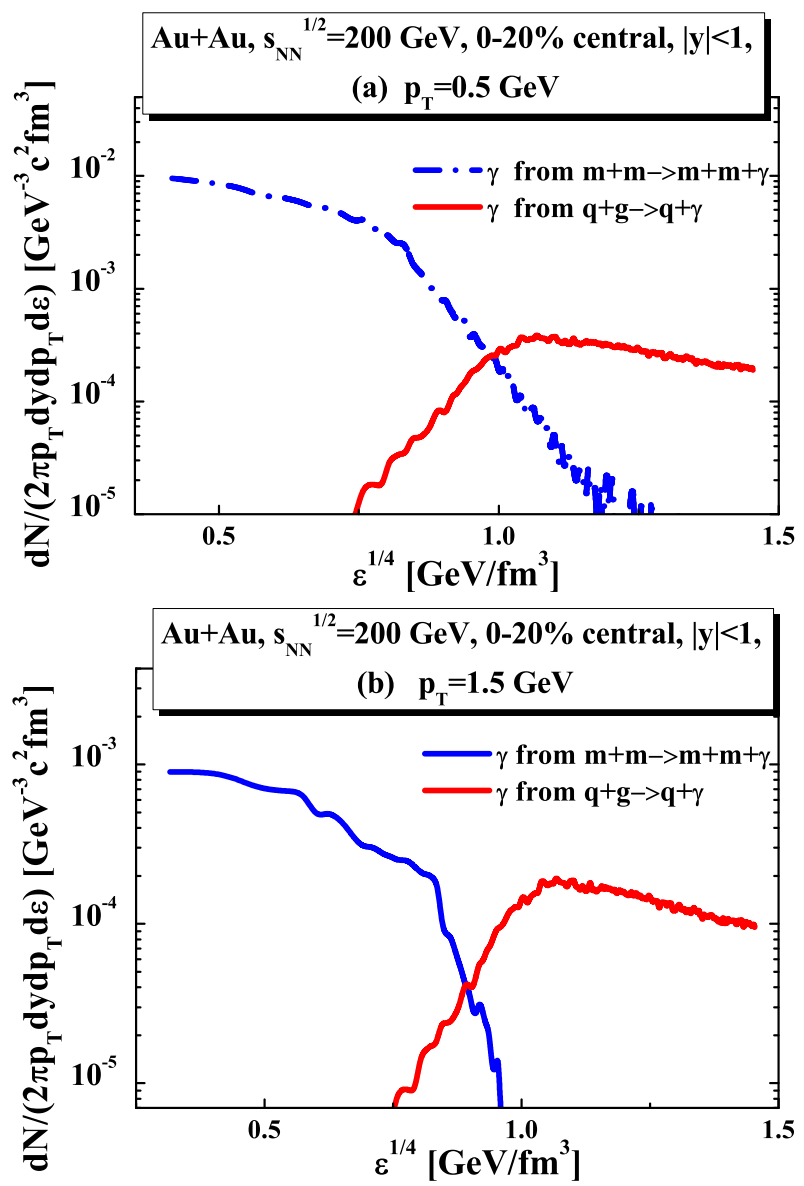

FIG. 13: (Color on-line) Top: Yield of photons with the transverse momentum $p_{T}=0.5 \mathrm{GeV}$ at mid-rapidity produced in 0-20\% most central $A u+A u$ collisions as functions of the approximate local "temperature" (the fourth-root of the energy density) from the PHSD from meson-meson bremsstrahlung (dash-dotted lines) and gluon Compton scattering (solid lines). Bottom: Same as in the top panel for photons with the transverse momentum $p_{T}=1.5 \mathrm{GeV}$.

times over which the produced hadrons continue to interact elastically, which is accompanied by the photon bremsstrahlung in case of charged hadrons.

We now increase the invariant energy $\sqrt{s_{N N}}$ by roughly another factor of 14 . In Fig. 14 we show the direct photon yield from PHSD in $\mathrm{Pb}+\mathrm{Pb}$ collisions at the invariant energy $\sqrt{s_{N N}}=2.76 \mathrm{TeV}$ for $0-40 \%$ centrality. When comparing the theoretical calculations to the preliminary data of the ALICE Collaboration from Ref. 2], we find a rather good overall agreement with the data within about a factor of 2 in the range of transverse momenta $p_{T}$ from 1 to $4 \mathrm{GeV}$. On the other hand, the calculations tend to underestimate the preliminary data in the low- $p_{T}$ region [99].

Furthermore, we provide predictions for the centralitydependence of the direct photon transverse momentum distributions at the LHC energy in Fig. 15], since the differential investigation in centralities and energies of the

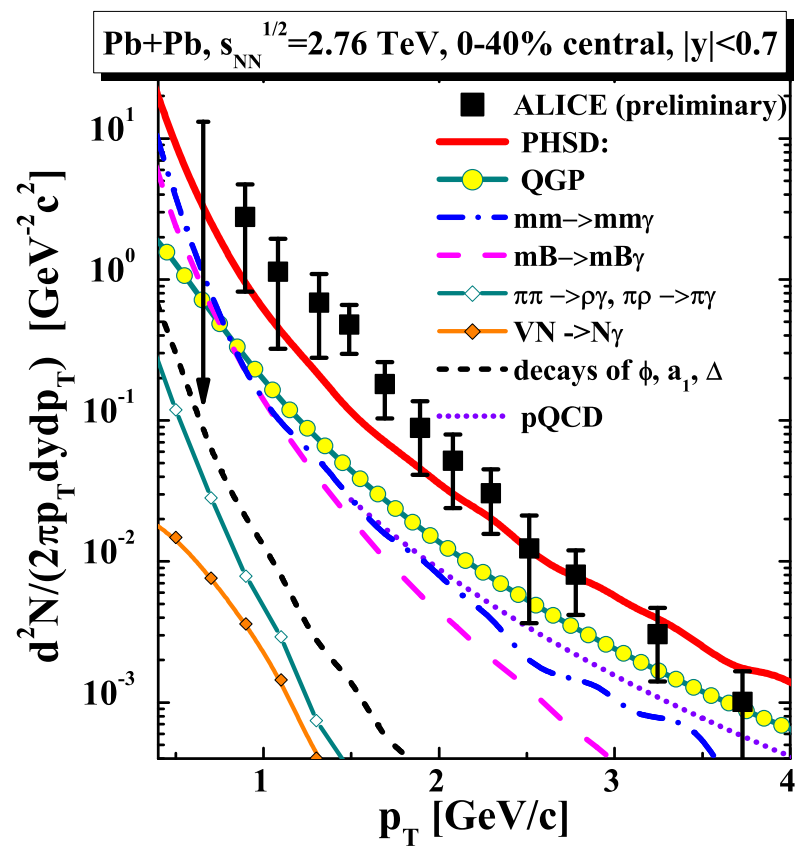

FIG. 14: (Color on-line) Yield of direct photons at midrapidity in $\mathrm{Pb}+\mathrm{Pb}$ collisions at the invariant energy $\sqrt{s_{N N}}=$ $2.76 \mathrm{TeV}$ for $0-40 \%$ centrality within the PHSD in comparison to the preliminary data from the ALICE Collaboration [2].

heavy-ion collisions will provide crucial information for a clarification of the relative importance of the contributing photon sources. Very recently, the data of the ALICE Collaboration for the photon yield at these three centralities has become available [98], we show them as the black symbols in Fig. 15.

In conclusion, we have found that from SPS to LHC energies the radiation from the SQGP constitutes less than half of the observed number of direct photons for central reactions. The radiation from hadrons and their interaction - which are not measured separately so far - give a considerable contribution especially at low transverse momentum. The dominant hadronic sources are the meson decays, the meson-meson bremsstrahlung and the meson-baryon bremsstrahlung. While the former (e.g. the decays of $\omega, \eta^{\prime}, \phi$ and $a_{1}$ mesons) can be subtracted from the spectra once the mesonic yields are determined independently by experiment, the reactions $\pi+\rho \rightarrow \pi+\gamma$, $\pi+\pi \rightarrow \rho+\gamma, V+N \rightarrow N+\gamma, \Delta \rightarrow N+\gamma$ as well as the meson-meson and meson-baryon bremsstrahlung can be separated from the partonic sources only with the assistance of theoretical models.

\section{B. Elliptic flow of direct photons}

The azimuthal momentum distribution of the emitted particles is commonly expressed in the form of a Fourier 

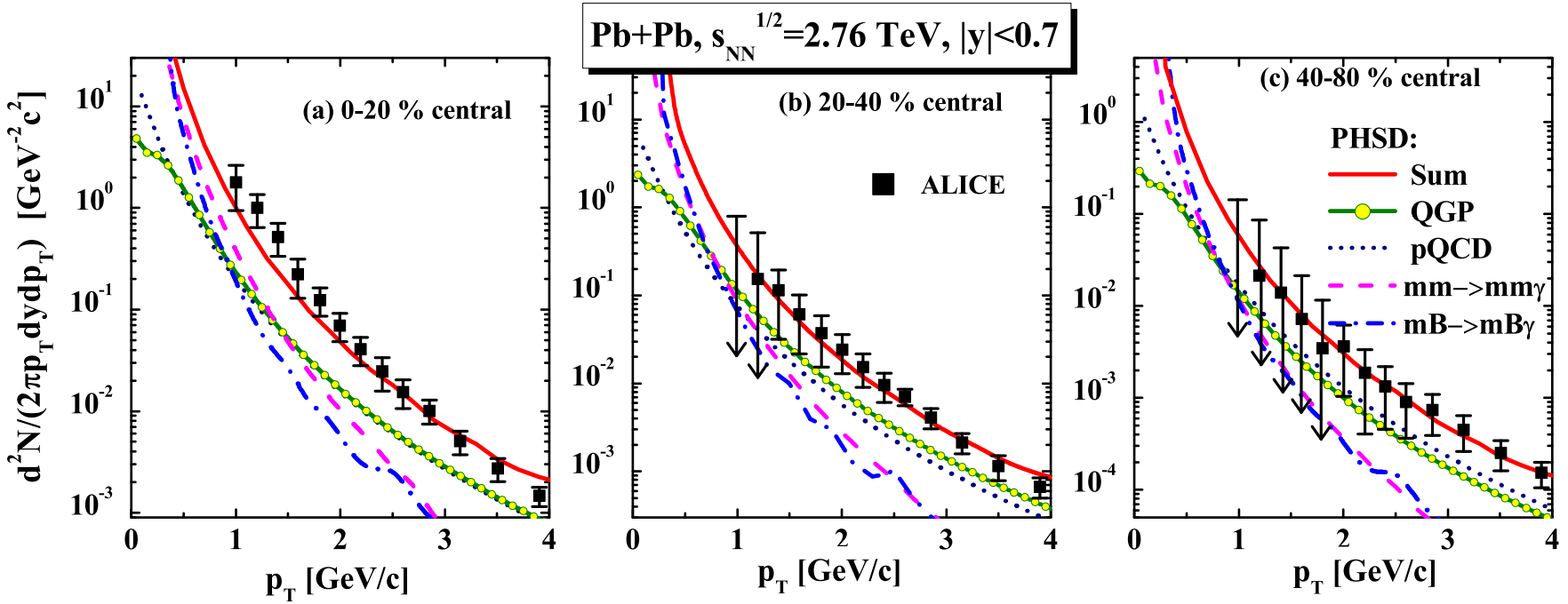

FIG. 15: (Color on-line) Transverse momentum spectra of direct photons at mid-rapidity in $\mathrm{Pb}+\mathrm{Pb}$ collisions at the invariant energy $\sqrt{s_{N N}}=2.76 \mathrm{TeV}$ for the three centrality bins $-0-20 \%, 20-40 \%$ and $40-80 \%-$ as predicted within the PHSD. The black symbols show the very recently available data from the ALICE Collaboration [98]. See the legend for the individual contributions.

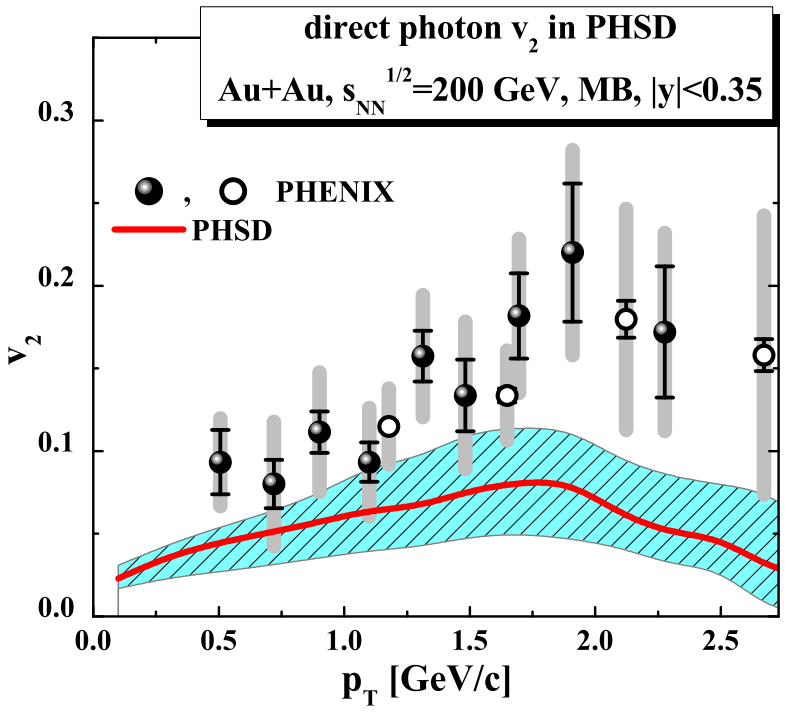

FIG. 16: (Color on-line) Elliptic flow $v_{2}$ versus transverse momentum $p_{T}$ for the direct photons produced in minimum bias $\mathrm{Au}+\mathrm{Au}$ collisions at $\sqrt{s_{N N}}=200 \mathrm{GeV}$ calculated within the PHSD (solid red line); the blue band reflects the uncertainty in the modeling of the cross sections for the individual channels. The data of the PHENIX Collaboration are from Ref. [1].

series as

$E \frac{d^{3} N}{d^{3} p}=\frac{d^{2} N}{2 \pi p_{T} d p_{T} d y}\left(1+\sum_{n=1}^{\infty} 2 v_{n}\left(p_{T}\right) \cos \left[n\left(\psi-\Psi_{n}\right)\right]\right)$,

where $v_{n}$ is the magnitude of the $n^{\prime}$ th order harmonic term relative to the angle of the initial-state spatial plane of symmetry $\Psi_{n}$ and $p=(E, \vec{p})$ is the four-momentum of the particle under consideration. We here focus on the coefficients $v_{2}$ and $v_{3}$ which implies that we have to perform event by event calculations in order to catch the initial fluctuations in the shape of the interaction zone and the event plane $\Psi_{E P}$. We calculate the triangular flow $v_{3}$ with respect to $\Psi_{3}$ as $v_{3}\left\{\Psi_{3}\right\}=\langle\cos (3[\psi-$ $\left.\left.\left.\Psi_{3}\right]\right)\right\rangle / \operatorname{Res}\left(\Psi_{3}\right)$. The event plane angle $\Psi_{3}$ and its resolution $\operatorname{Res}\left(\Psi_{3}\right)$ are evaluated using the hadron-hadron correlations at larger rapidities as described in Ref. [100] via the two-sub-events method [101, 102].

We recall that the second flow coefficient $v_{2}$ carries information on the interaction strength in the system and thus on the state of matter and its properties - at the space-time point, from which the measured particles are emitted. The elliptic flow $v_{2}$ reflects the azimuthal asymmetry in the momentum distribution of the produced particles ( $p_{x}$ vs $\left.p_{y}\right)$, which is a consequence of the geometrical azimuthal asymmetry of the initial reaction region. If the produced system is a weakly-interacting gas, then the initial spatial asymmetry is not effectively transferred into the final distribution of the momenta. On the contrary, if the produced matter has the properties of a liquid, then the initial geometrical configuration is reflected in the final particle momentum distribution.

More than a decade ago, the WA98 Collaboration has measured the elliptic flow $v_{2}$ of photons produced in $P b+P b$ collisions at the beam energy of $E_{\text {beam }}=$ $158 \mathrm{AGeV}$ [104], and it was found that the $v_{2}\left(\gamma^{\text {incl }}\right)$ of the low-transverse-momentum inclusive photons was equal to the $v_{2}\left(\gamma^{\pi}\right)$ of pions within the experimental uncertainties. This observation has lead to the conclusion that either (Scenario 1:) the elliptic flow of the direct photons was comparable in magnitude to the $v_{2}\left(\gamma^{\text {incl }}\right)$ and $v_{2}\left(\gamma^{\text {decay }}\right)$, or (Scenario 2:) the contribution of the 


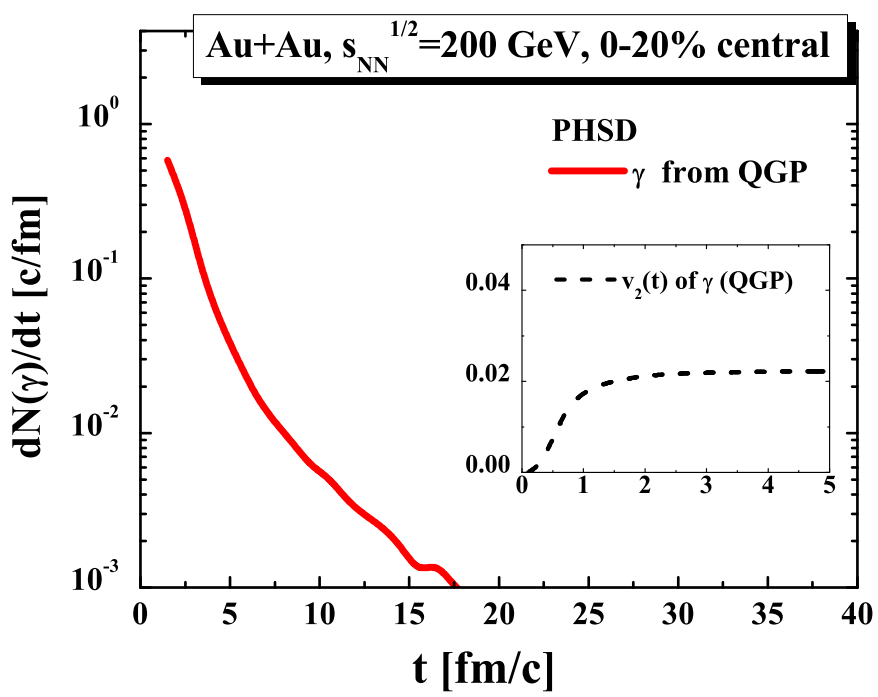

FIG. 17: (Color on-line) Rate of photon production in the QGP versus time for $0-20 \% \mathrm{Au}+\mathrm{Au}$ collisions at $\sqrt{s_{N N}}=200$ $\mathrm{GeV}$ from the PHSD. The insert shows the time evolution of the elliptic flow of photons from the partonic sources.

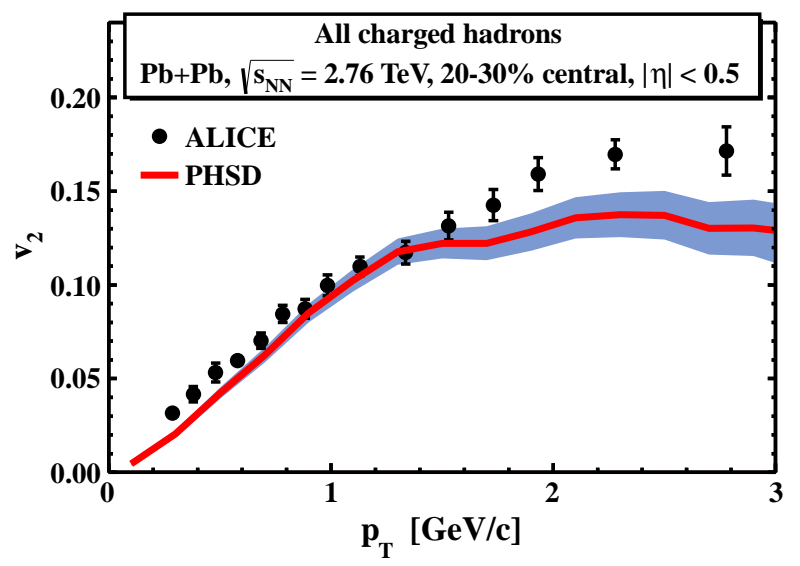

FIG. 18: (Color on-line) Elliptic flow $v_{2}$ versus transverse momentum $p_{T}$ for the charged particles produced in $20-30 \%$ central $\mathrm{Pb}+\mathrm{Pb}$ collisions at $\sqrt{s_{N N}}=2.76 \mathrm{TeV}$ from the PHSD (solid red line) in comparison to the data from the ALICE Collaboration [103]; the blue band reflects the statistical uncertainty of the PHSD calculations.

direct photons to the inclusive ones was negligible. However, the photon spectrum measured by the WA98 Collaboration showed a significant yield of direct photons at low transverse momentum. Thus the scenario 2 can be ruled out. Consequently, one has to assume that the observed direct photons of low $p_{T}$ had a significant elliptic anisotropy $v_{2}$ - of the same order of magnitude as the hadronic flow. The interpretation [41, 65] of the low- $p_{T}$ direct photon yield measured by WA98 as dominantly produced by the bremsstrahlung process in the mesonic

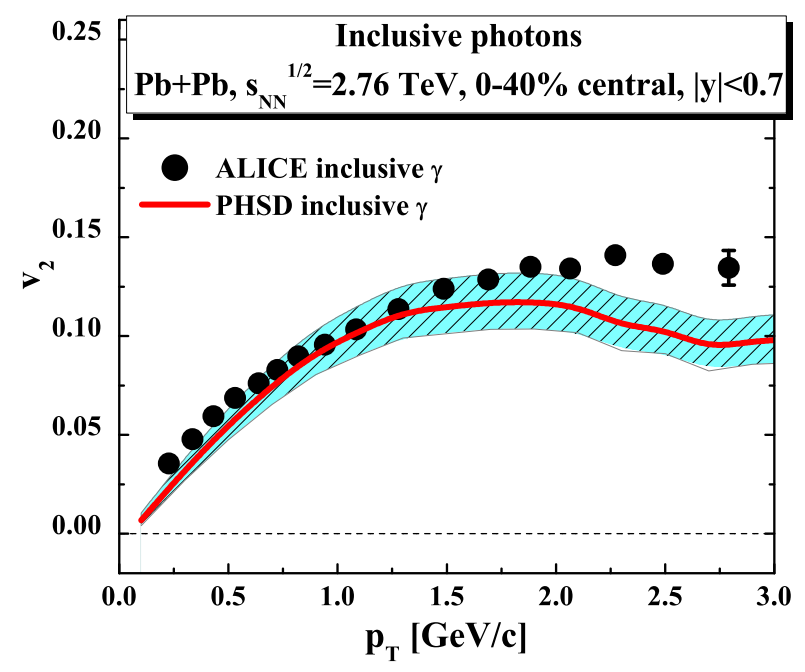

FIG. 19: (Color on-line) Elliptic flow $v_{2}$ versus transverse momentum $p_{T}$ for the inclusive photons produced in $0-40 \%$ central $\mathrm{Pb}+\mathrm{Pb}$ collisions at $\sqrt{s_{N N}}=2.76 \mathrm{TeV}$ from the PHSD (solid red line) in comparison to the data from the ALICE Collaboration [33]; the blue error band reflects the finite statistics and the uncertainty in the modeling of the cross sections for the individual channels.

collisions $\pi+\pi \rightarrow \pi+\pi+\gamma$ is in accord with the WA98 data on the inclusive photon $v_{2}\left(\gamma^{i n c l}\right)$.

Let us note that the same conclusions apply also to the most recent studies of the photon elliptic flow at RHIC and LHC. The PHENIX and ALICE Collaborations have measured the inclusive photon $v_{2}$ and found that at low transverse momenta it is comparable to the $v_{2}\left(p_{T}\right)$ of decay photons as calculated in cocktail simulations based on the known mesonic $v_{2}\left(p_{T}\right)$. Therefore (a) either the yield of the direct photons to the inclusive ones is not statistically significant in comparison to the decay photons or (b) the elliptic flow of the direct photons must be as large as $v_{2}\left(\gamma^{\text {decay }}\right)$ and $v_{2}\left(\gamma^{\text {incl }}\right)$.

In Refs. [6, 7] we have shown the elliptic flow of the inclusive and direct photons produced in minimum bias $\mathrm{Au}+\mathrm{Au}$ collisions at $\sqrt{s_{N N}}=200 \mathrm{GeV}$ from the PHSD in comparison to the data of the PHENIX Collaboration. We found that the data on the inclusive photon $v_{2}$ could approximately be described. Furthermore, the pion decay photons dominate the inclusive photon spectrum. Since the elliptic flow of pions is under control in PHSD in comparison to the data from the PHENIX and STAR Collaborations (cf. Refs. 1, 6, 47, 105, 106]), the spectrum of decay photons is also predicted reliably by the model. However, the good agreement with the inclusive photon spectrum is especially meaningful due to the good description of the direct photon spectrum which was presented above in section IV]

After subtracting the contribution of the decay photons from the inclusive photons, the direct photon $v_{2}$ 


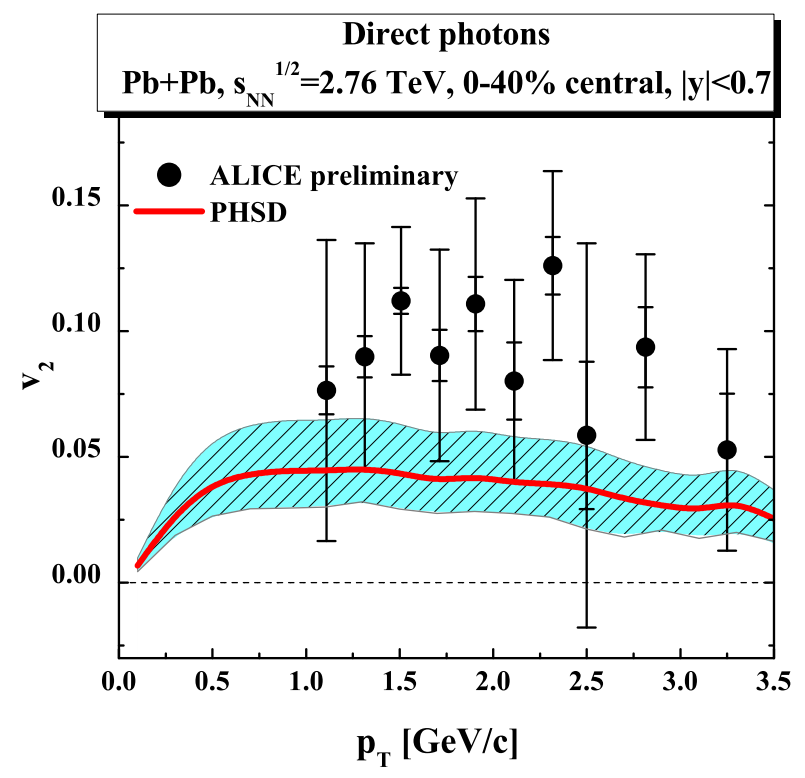

FIG. 20: (Color on-line) Elliptic flow $v_{2}$ versus transverse momentum $p_{T}$ for the direct photons produced in $0-40 \%$ central $\mathrm{Pb}+\mathrm{Pb}$ collisions at $\sqrt{s_{N N}}=2.76 \mathrm{TeV}$ from the PHSD (solid red line) in comparison to the preliminary data from the ALICE Collaboration 33]; the blue error band is dominated by the uncertainty in the modeling of the cross sections for the individual channels.

is accessed experimentally. In the PHSD, we calculate the direct photon $v_{2}\left(\gamma^{d i r}\right)$ by building the weighted sum of the channels, which are not subtracted by the datadriven methods, as follows: the photons from the quarkgluon plasma, from the initial hard parton collisions (pQCD photons), from the decays of short-living resonances ( $a_{1}$-meson, $\phi$-meson, $\Delta$-baryon), from the binary meson + meson and meson+baryon channels $(\pi+\rho \rightarrow$ $\pi+\gamma, \pi+\pi \rightarrow \rho+\gamma, V+p / n \rightarrow n / p+\gamma)$, and from the bremsstrahlung in the elastic meson+meson and meson+baryon collisions $(m+m \rightarrow m+m+\gamma$, $m+B \rightarrow m+B+\gamma)$. We calculate the direct photon $v_{2}$ by summing up the elliptic flow of the individual channels contributing to the direct photons, using their contributions to the spectrum as the relative $p_{T}$-dependent weights, $w_{i}\left(p_{T}\right)$, i.e.

$$
v_{2}\left(\gamma^{d i r}\right)=\sum_{i} v_{2}\left(\gamma^{i}\right) w_{i}\left(p_{T}\right)=\frac{\sum_{i} v_{2}\left(\gamma^{i}\right) N_{i}\left(p_{T}\right)}{\sum_{i} N_{i}\left(p_{T}\right)}
$$

The results for the elliptic flow $v_{2}\left(p_{T}\right)$ of direct photons produced in $A u+A u$ collisions at the top RHIC energy are shown in Fig. 16. In comparison to the previous results within the PHSD approach [6, 7], the elliptic flow in the intermediate region of the transverse momenta $1.0<p_{T}<2.0 \mathrm{GeV}$ is reduced by about $50 \%$ due to the modifications in our computation of the bremsstrahlung channels beyond the soft-photon approximation in the present work. According to our calculations of the direct photon spectra (as presented above), almost half of the direct photons measured by PHENIX (in central collisions) stems from the collisions of quarks and gluons in the deconfined medium created in the initial phase of the collision. The photons produced in the QGP carry a very small $v_{2}$ and lead to an overall direct photon $v_{2}$ about a factor of 2 below the pion $v_{2}(\pi)$ even though the other channels in the sum (43) have large elliptic flow coefficients $v_{2}$ of the order of $v_{2}(\pi)$ (cf. Fig. 7 of Ref. [6]).

Indeed, the parton collisions - producing photons in the QGP - take place throughout the evolution of the collision but the collision rate falls rapidly with time and thus the production of photons from the QGP is dominated by the early times. We illustrate this in Fig 17. where it is seen that the rate of photon emission $d N_{\gamma} / d t$ from QGP sources drops by orders of magnitude after the first $10 \mathrm{fm} / \mathrm{c}$. As a consequence, the elliptic flow 'picked up' by the photons from the parent parton collisions saturates after about a few $\mathrm{fm} / \mathrm{c}$ and reaches a relatively low value of about 0.02 , only. We note that a delayed production of charges from the strong gluon fields ('glasma' 108 111]) might shift the QGP photon production to somewhat later times when the elliptic flow is built up more. However, we cannot quantitatively answer whether the additional evolution in the pre-plasma state could generate considerable additional direct photon $v_{2}$.

In the following we present calculations/predictions for the elliptic flow of inclusive and direct photons produced in $P b+P b$ collisions at the energy of $\sqrt{s_{N N}}=2.76 \mathrm{TeV}$. First, we show in Fig. 18 the elliptic flow $v_{2}$ versus transverse momentum $p_{T}$ for the charged hadrons produced in $20-30 \%$ central $\mathrm{Pb}+\mathrm{Pb}$ collisions at $\sqrt{s_{N N}}=2.76 \mathrm{TeV}$ from the PHSD (solid red line); the blue band reflects the statistical uncertainty of the calculations. The PHSD results are compared to the data of the ALICE Collaboration [103] which suggests that the bulk dynamics is reasonably under control in the PHSD at these energies and the elliptic flow of the final charged particles is described up to $p_{T} \approx 2 \mathrm{GeV} / \mathrm{c}$ in this centrality range.

We, furthermore, present our calculations/predictions for the elliptic flow of inclusive and direct photons produced in $P b+P b$ collisions at the energy of $\sqrt{s_{N N}}=$ $2.76 \mathrm{TeV}$ at the LHC within the acceptance of the ALICE detector. Fig. 19] shows PHSD calculations for the elliptic flow $v_{2}$ versus transverse momentum $p_{T}$ for the inclusive photons produced in $0-40 \%$ central $\mathrm{Pb}+\mathrm{Pb}$ collisions at $\sqrt{s_{N N}}=2.76 \mathrm{TeV}$ (solid red line) with the blue error band reflecting the finite statistics and the theoretical uncertainty in the modeling of the cross sections. A comparison to the respective data from the ALICE Collaboration [33] shows a comparable agreement as in case of the $v_{2}$ for the charged hadrons.

The elliptic flow $v_{2}\left(p_{T}\right)$ of direct photons produced in $0-40 \%$ central $\mathrm{Pb}+\mathrm{Pb}$ collisions at $\sqrt{s_{N N}}=2.76 \mathrm{TeV}$ from the PHSD (solid red line) is shown in Fig. 20 in comparison to the preliminary data from the ALICE Collaboration [33]; the blue error band is again dominated by the uncertainty in the modeling of the cross sections 


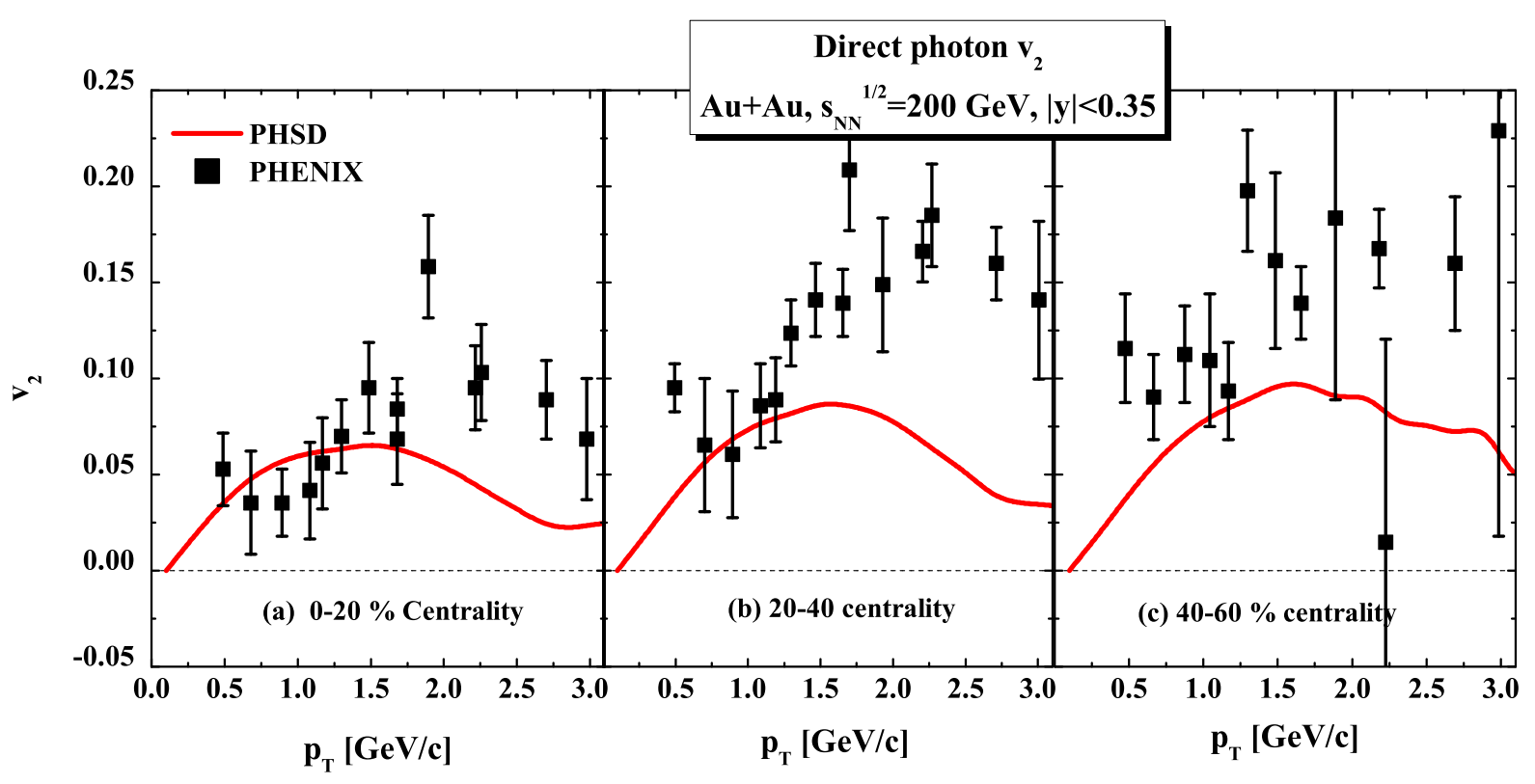

FIG. 21: Centrality dependence of the direct photon $v_{2}$ for $\mathrm{Au}+\mathrm{Au}$ collisions at $\sqrt{s_{N N}}=200 \mathrm{GeV}$ for different centralities (see legend); the data from the PHENIX Collaboration [31, 107] are compared to the earlier PHSD predictions from Ref. 7].

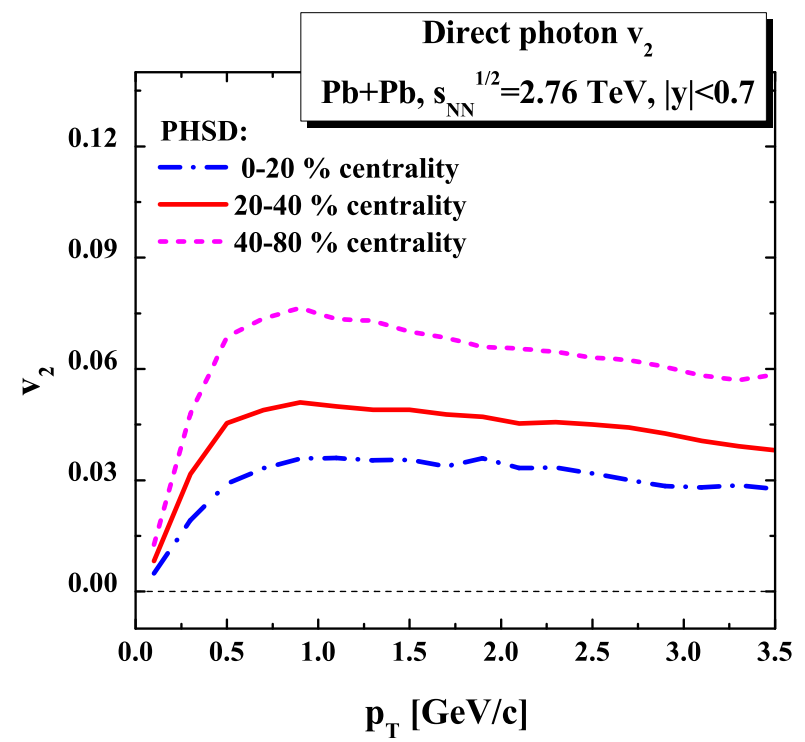

FIG. 22: (Color on-line) Elliptic flow $v_{2}$ of direct photons from $\mathrm{PHSD}$ versus transverse momentum $p_{T}$ produced in $\mathrm{Pb}+\mathrm{Pb}$ collisions at $\sqrt{s_{N N}}=2.76 \mathrm{TeV}$ for different centrality classes (see legend).

for the individual channels. As in case of $\mathrm{Au}+\mathrm{Au}$ collisions at the top RHIC energy we slightly underestimate the direct photon $v_{2}$, however, new data with higher accuracy will be needed to shed further light on the direct photon $v_{2}$ puzzle.

The centrality dependence of the direct photon elliptic flow has been calculated within the PHSD in Ref. [7] for $\mathrm{Au}+\mathrm{Au}$ collisions at the top RHIC energy. Fig. 21 presents a direct comparison of the PHSD predictions for $v_{2}\left(p_{T}\right)$ from Ref. [7] for $\mathrm{Au}+\mathrm{Au}$ collisions at $\sqrt{s_{N N}}=200$ $\mathrm{GeV}$ in the centrality classes $0-20 \%$ (a), 20-40\% (b) and $40-60 \%$ (c) with the data from Refs. [30, 31]. Whereas the elliptic flow is roughly described in the most central class there is an increasing tendency to underestimate in the PHSD the strong elliptic flow especially for peripheral collisions where some additional source might be present. Thus the observed centrality dependence of the elliptic flow is roughly in agreement with the interpretation that a large fraction of the direct photons is of hadronic origin (in particular from the bremsstrahlung in meson+meson and meson+baryon collisions); the latter contribution becomes stronger in more peripheral collisions. But more precise data will be mandatory for a robust conclusion.

We close this Subsection in providing predictions for the centrality dependence of the direct photon $v_{2}\left(p_{T}\right)$ in $\mathrm{Pb}+\mathrm{Pb}$ collisions at $\sqrt{s_{N N}}=2.76 \mathrm{TeV}$ in the centrality classes $0-20 \%, 20-40 \%$ and $40-80 \%$ which are of relevance for the upcoming measurements by the ALICE Collaboration at the LHC. The actual results from PHSD are displayed in Fig. 22 and show a very similar centrality dependence as in case of $\mathrm{Au}+\mathrm{Au}$ collisions at the top RHIC energy.

\section{Triangular flow of direct photons}

We have seen in the previous sections that the measured spectra of direct photons could be reproduced by the PHSD calculations within a factor of 2 (which is comparable with the current accuracy of the measurements). 


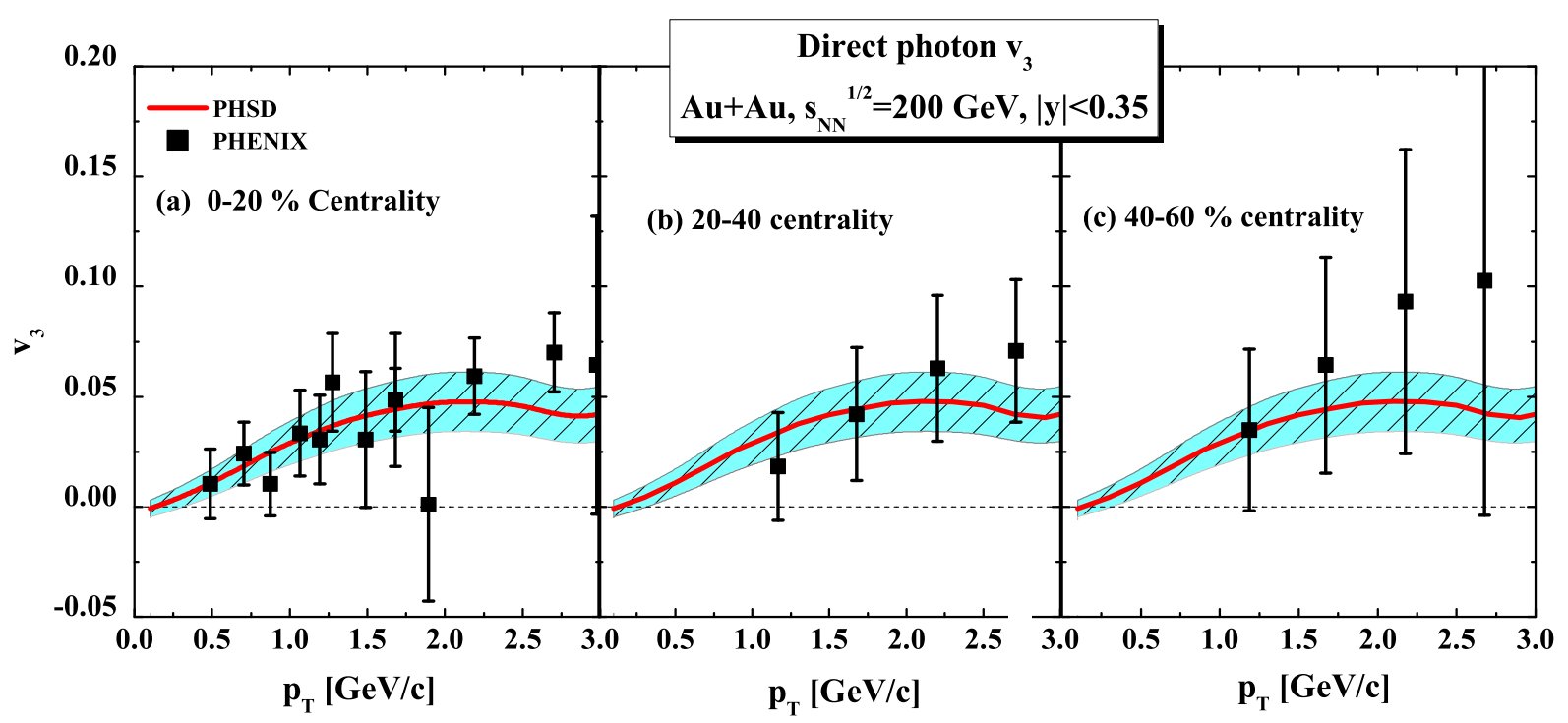

FIG. 23: (Color on-line) Triangular flow $v_{3}$ versus transverse momentum $p_{T}$ for the direct photons produced in Au+Au collisions at $\sqrt{s_{N N}}=200 \mathrm{GeV}$ in three centrality classes (see legends). The PHSD results are shown by the solid red lines in comparison to the data of the PHENIX Collaboration (black symbols) taken from Ref. [107, 112].

Also, the inclusive photon $v_{2}$ was well described and the elliptic flow of direct photons was qualitatively in line with the data (within a factor of 2) and attributed essentially to hadronic sources.

On the other hand, there exists an alternative interpretation of the strong elliptic flow of direct photons, in which the azimuthal asymmetry of the photons is due to the initial strong magnetic field essentially produced by spectator charges (protons). Indeed, the magnetic field strength in the very early reaction stage reaches up to $e B_{y} \approx 5 m_{\pi}^{2}$ in semi-peripheral $A u+A u$ collisions at $\sqrt{s_{N N}}=200 \mathrm{GeV}$ (see the calculations within the PHSD in Ref. 113]; comparable estimates have been obtained also in Refs. 114, 115]). These strong magnetic fields might influence the photon production via the polarization of the medium, e.g. by influencing the motion of charged quarks in the QGP, or by directly inducing a real photon radiation via the virtual photon ( $\vec{B}$-field) coupling to a quark loop and (multiple) gluons; the photons are then produced azimuthal asymmetrically.

The photon production under the influence of strong magnetic fields has been calculated in Refs. [19, 25, 114, 116]. The observed spectra and elliptic flow of direct photons could be explained using suitable assumptions on the conductivity, bulk viscosity or degree of chemical equilibration in the early produced matter. The common feature of these calculations was that the triangular flow coefficient $v_{3}$ of the direct photons was expected to be very small. Indeed, the magnetic field may lead to an azimuthal asymmetry $v_{2}$ but not to a triangular mode.

Consequently, it is of interest to measure experimentally the third flow coefficient $v_{3}\left(p_{T}\right)$ and to compare it to the calculations in the different classes of models: (a) those attributing the large elliptic flow and strong yield

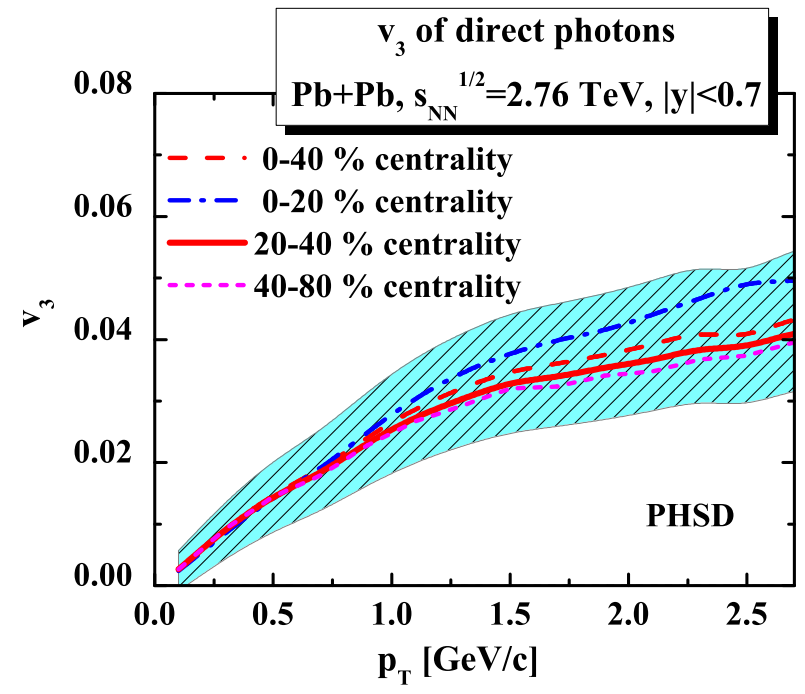

FIG. 24: (Color on-line) Triangular flow $v_{3}$ versus transverse momentum $p_{T}$ for the direct photons produced in different centrality classes for $\mathrm{Pb}+\mathrm{Pb}$ collisions at $\sqrt{s_{N N}}=2.76 \mathrm{TeV}$ from the PHSD (see legend); the blue band reflects the uncertainty in the modeling of the cross sections for the individual channels and give a measure of the present level of accuracy.

of direct photons to hadronic sources, e.g. the PHSD transport approach; (b) the models suggesting the large azimuthal asymmetry and additional yield of direct photons to be caused by the early magnetic fields; (c) the models assuming that the yield of direct photons at low $p_{T}$ is dominated by partonic channels.

In Fig. 23 we present our results for the triangular flow $v_{3}$ versus transverse momentum $p_{T}$ for the direct photons produced in $\mathrm{Au}+\mathrm{Au}$ collisions at $\sqrt{s_{N N}}=200 \mathrm{GeV}$ 
from the PHSD (solid red lines) for 0-20\% (a), 20-40\% (b) and 40-60\% (c) centrality. The PHSD gives a positive non-zero triangular flow of direct photons up to $6 \%$ with very little centrality dependence on the level of the present accuracy $(\sim 25 \%)$. The PHSD results are in agreement with the data of the PHENIX Collaboration from Refs. [112, 117, 118] which suggests that the scenario (a) is compatible with the measurements.

Accordingly, the data from PHENIX in Fig. 23] do not point towards an interpretation of the direct photons being dominantly produced in the early stage under the influence of the magnetic field (b), because the $v_{3}$ of these photons is expected to be close to zero. Of course, the photon production in the magnetic fields occurs on top of other channels, which may carry finite $v_{3}$. But the weighted sum of all the channels including the magneticfield-induced photons will give a smaller $v_{3} \neq 0$ than the sum without this channel. The scenario (c) has been studied by other groups within a hydrodynamic modeling of the collision in Refs. [15, 119]. The triangular flow $v_{3}\left(p_{T}\right)$ of direct photons from Refs. [15, 119] is about a factor of 2 smaller than that obtained in the present work from the PHSD. It will be possible to differentiate between the scenarios in the future, when data of higher accuracy will become available.

We finally show in Fig. 24 our predictions for the triangular flow $v_{3}\left(p_{T}\right)$ of direct photons produced in $\mathrm{Pb}+\mathrm{Pb}$ collisions at $\sqrt{s_{N N}}=2.76 \mathrm{TeV}$ for different centralities as will be measured at the LHC. We expect the triangular flow of direct photons to reach at maximum $v_{3}^{\max }=0.04 \pm 0.015$, thus being comparable to the charged hadron $v_{3}$ [47]. The centrality dependence of $v_{3}\left(p_{T}\right)$ turns out to be low and is practically constant within the accuracy of the PHSD calculations. An experimental confirmation of this expectation could further affirm the notion of large hadronic contributions to the direct photons and in particular the photon production via the bremsstrahlung in meson and baryon collisions.

\section{SUMMARY}

In the present work we have calculated the transverse momentum spectra, the elliptic flow $v_{2}$ and triangular flow $v_{3}$ of direct photons produced in $\mathrm{Au}+\mathrm{Au}$ collisions at $\sqrt{s_{N N}}=200 \mathrm{GeV}$ and in $\mathrm{Pb}+\mathrm{Pb}$ collisions at $\sqrt{s_{N N}}=2.76 \mathrm{TeV}$ using the microscopic PHSD transport approach. For thermal photon production we have considered the interactions of quarks and gluons in the strongly interacting quark-gluon plasma (sQGP) $(q+\bar{q} \rightarrow g+\gamma$ and $q(\bar{q})+g \rightarrow q(\bar{q})+\gamma)$, the photon production in the hadronic decays $(\pi \rightarrow \gamma+\gamma$, $\eta \rightarrow \gamma+\gamma, \omega \rightarrow \pi+\gamma, \eta^{\prime} \rightarrow \rho+\gamma, \phi \rightarrow \eta+\gamma$, $\left.a_{1} \rightarrow \pi+\gamma, \Delta \rightarrow N+\gamma\right)$ as well as the interactions $(\pi+\pi \rightarrow \rho+\gamma, V+\pi \rightarrow \pi+\gamma$, the $2 \rightarrow 2$ interaction of mesons and baryons $\rho+n / p \rightarrow \gamma+n / p$, and last but not least the bremsstrahlung radiation in meson + meson and meson+baryon scattering $m+m / B \rightarrow m+m / B+\gamma$ throughout the evolution of the collision. The pQCD photons produced in the initial hard binary scatterings are added to the thermal photons in order to obtain the final direct photon spectrum.

In extension of our previous works on photon production in heavy-ion collisions at the top RHIC energy we

(i) went beyond the soft-photon approximation (SPA) in the calculation of the bremsstrahlung processes meson + meson $\rightarrow$ meson + meson $+\gamma$, meson + baryon $\rightarrow$ meson + baryon $+\gamma$,

(ii) quantified the suppression at low $p_{T}$ due the LandauPomeranchuk-Migdal (LPM) effect in connection with the electric conductivity, and

(iii) incorporated the $V+N \rightarrow \gamma+N$ and $\Delta \rightarrow N+\gamma$ channels into the PHSD. Furthermore,

(iv) have presented calculations for $\mathrm{Pb}+\mathrm{Pb}$ collisions at SPS and LHC energies.

The result from Ref. [65] for the reaction $\pi+\pi \rightarrow$ $\pi+\pi+\gamma$, obtained within the one-boson exchange model beyond the soft-photon approximation - available up to the photon energy of $0.4 \mathrm{GeV}$ - is confirmed by our present calculations that extend to $2 \mathrm{GeV}$. Furthermore, the improved SPA (28) gives a very good approximation to the exact result even at high $\sqrt{s}$ as long as the model for the elastic cross section is sufficiently realistic. In comparison, the constant-cross-section approximation based on the formula (30) (used before) overestimates the exact rates for $q_{0}>1 \mathrm{GeV}$ and underestimates for $q_{0}<0.4 \mathrm{GeV}$. The OBE differential cross sections have been implemented in the PHSD transport approach for photon production studies in heavy-ion reactions from SPS to LHC energies.

Furthermore, we have given an estimate for the photon suppression due to the Landau-Pomeranchuk-Migdal (LPM) effect employing the approximation (36) that captures the relevant physics. When inserting the interaction time of the partons from PHSD at finite temperature we obtain a photon rate that is well in line with Eq. (37) in the limit of vanishing photon energy. We recall that the electric conductivity $\sigma_{0}$ regulates the divergence of the photon rate and has been evaluated in the PHSD in Ref. [77] in rough agreement with related results from lattice QCD. The actual results in Fig. 9 demonstrate that the LPM effect becomes important for photon energies below $0.4 \mathrm{GeV}$ in case of dense and strongly interacting partons. Since the experimental photon spectra measured so far at RHIC and LHC energies start from higher phton energies the LPM effect might be safely discarded.

In case of relativistic heavy-ion collisions we have found that the PHSD calculations reproduce the transverse momentum spectra of direct photons as measured by the PHENIX Collaboration in Refs. [29, 30] for $\mathrm{Au}+\mathrm{Au}$ collisions at the top RHIC energy. The calculations reveal the channel decomposition of the observed direct photon spectrum and show that the photons produced in the QGP constitute at most about $50 \%$ of the direct photons in central collisions with the rest being distributed among 
the other channels: mesonic interactions, decays of massive hadronic resonances and the initial hard scatterings. Our calculations demonstrate that the photon production in the QGP is dominated by the early phase (similar to hydrodynamic models) and is localized in the center of the fireball, where the collective flow is still rather low, i.e. on the $2-3 \%$ level, only. Thus, the strong $v_{2}$ of direct photons - which is comparable to the hadronic $v_{2}$ - in PHSD is attributed to hadronic channels, i.e. to meson and baryon induced reactions. On the other hand, the strong $v_{2}$ of the 'parent' hadrons, in turn, stems from the interactions in the QGP via collisions and the partonic mean-field potentials. Accordingly, the presence of the QGP shows up 'indirectly' in the direct photon elliptic flow.

We, furthermore, have demonstrated that the elliptic flow of charged hadrons from PHSD is in a reasonable agreement with the ALICE data. Predictions/calculations for the inclusive photon $v_{2}$ and direct photon $v_{2}$ have been provided as well as associated results for the triangular direct photon flow $v_{3}\left(p_{T}\right)$ which is as large as the charged hadron $v_{3}$ at $\sqrt{s_{N N}}=200 \mathrm{GeV}$ and $\sqrt{s_{N N}}=2.76 \mathrm{TeV}$. The large triangular flow of direct photons seen by PHENIX at the top RHIC energy is in line with the PHSD calculations and in conflict with a scenario that attributes the large direct photon $v_{2}$ to the influence of the early strong magnetic field from the spectator charges.

The centrality dependence of the direct photon yield and flow has the potential to further clarify the direct photon production mechanisms. We find a good agreement between the PHENIX measurements and the PHSD calculations at the top RHIC energy. In particular, the integrated thermal photon yield in PHSD was predicted to scale as $N_{\text {part }}^{\alpha}$ with the exponent $\alpha \approx 1.5$, which is in a good agreement with the most recent measurement of $\alpha=1.48 \pm 0.08 \pm 0.04$ by the PHENIX Collaboration [31]. This observation supports the conclusion that the low transverse momentum direct photons have a strong contribution from the binary hadronic photon production sources, such as the meson + meson and meson +baryon bremsstrahlung. It will be important to investigate experimentally the scaling of the direct photon yield and flows $v_{2}$ and $v_{3}$ with centrality also in $\mathrm{Pb}+\mathrm{Pb}$ collisions at the LHC energies for which we have provided explicit predictions from the PHSD.

\section{Acknowledgements}

We appreciate fruitful discussions with C. Gale, J. F. Paquet, C. M. Ko, D. Cabrera, H. Berrehrah, L. McLerran, K. Eskola, I. Tserruya, C. Klein-Boesing, R. Rapp, H. van Hess, J. Stachel, U. Heinz, I. Selyuzhenkov, G. David, K. Redlich, K. Reygers, C. Shen, A. Drees, B. Bannier and F. Bock. The contributions of J. F. Paquet, C. Gale, K. Eskola, I. Helenius and C. Klein-Boesing have been essential for constraining the pQCD photon yield. We especially thank C. Gale for enriching this work with his ideas and support during his stay at the Frankfurt Institute for Advanced Studies. Furthermore, the authors are grateful to K. Reygers, J. Stachel and the ExtreMe Matter Institute for conducting a stimulating and productive EMMI-Rapid-ReactionTask-Force meeting on the "Direct-Photon Flow Puzzle". Furthermore, we acknowledge financial support through the "HIC for FAIR" framework of the "LOEWE" program. The computational resources have been provided by the LOEWE-CSC as well as the SKYLLA cluster at the Univ. of Giessen.
[1] A. Adare et al. (PHENIX Collaboration), Phys. Rev. Lett. 109, 122302 (2012).

[2] M. Wilde (ALICE Collaboration), Nucl. Phys. A904, 573c (2013).

[3] E. V. Shuryak, Phys. Lett. B78, 150 (1978).

[4] C. Song, C. M. Ko, and C. Gale, Phys. Rev. D50, 1827 (1994).

[5] G.-Q. Li and C. Gale, Phys. Rev. C58, 2914 (1998).

[6] O. Linnyk et al., Phys. Rev. C88, 034904 (2013).

[7] O. Linnyk, W. Cassing, and E. Bratkovskaya, Phys. Rev. C89, 034908 (2014).

[8] R. Chatterjee et al., Phys. Rev. Lett. 96, 202302 (2006).

[9] F.-M. Liu, T. Hirano, K. Werner, and Y. Zhu, Nucl. Phys. A830, 587C (2009).

[10] M. Dion et al., J. Phys. G38, 124138 (2011).

[11] M. Dion et al., Phys. Rev. C84, 064901 (2011).

[12] R. Chatterjee et al., Phys. Rev. C 88, 034901 (2013).

[13] C. Shen, U. W. Heinz, J.-F. Paquet, and C. Gale, Phys. Rev. C89, 044910 (2014).

[14] K. Dusling, Nucl. Phys. A839, 70 (2010).
[15] C. Shen, U. W. Heinz, J.-F. Paquet, I. Kozlov, and C. Gale, Phys. Rev. C91, 024908 (2015).

[16] H. van Hees, M. He, and R. Rapp, Nucl. Phys. A933, 256 (2014).

[17] C. Shen, U. Heinz, J.-F. Paquet, and C. Gale, Nucl. Phys. A 932, 184 (2014).

[18] V. Goloviznin, A. Snigirev, and G. Zinovjev, JETP Lett. 61, 054906 (2013).

[19] L. McLerran and B. Schenke, Nucl. Phys. A929, 71 (2014).

[20] A. Monnai, Phys. Rev. C90, 021901 (2014).

[21] F.-M. Liu and S.-X. Liu, Phys. Rev. C89, 034906 (2014).

[22] G. A. Almasi and G. Wolf, arXiv:1407.2047 (2014), arXiv:1407.2047.

[23] V. Ozvenchuk, O. Linnyk, M. Gorenstein, E. Bratkovskaya, and W. Cassing, Phys. Rev. C87, 024901 (2013).

[24] V. Ozvenchuk, O. Linnyk, M. Gorenstein, E. Bratkovskaya, and W. Cassing, Phys. Rev. C87, 
064903 (2013).

[25] A. Bzdak and V. Skokov, Phys. Rev. Lett. 110, 192301 (2013).

[26] K. Tuchin, Phys. Rev. C91, 014902 (2015).

[27] K. Tuchin, Phys. Rev. C87, 024912 (2013).

[28] B. Müller, S.-Y. Wu, and D.-L. Yang (2013), arXiv:1308.6568.

[29] A. Adare et al. (PHENIX), Phys. Rev. C 81, 034911 (2010).

[30] A. Adare et al. (PHENIX Collaboration), Phys. Rev. Lett. 104, 132301 (2010).

[31] A. Adare et al. (PHENIX), Phys. Rev. C91, 064904 (2015).

[32] H. Eggers, R. Tabti, C. Gale, and K. Haglin, Phys. Rev. D53, 4822 (1996).

[33] D. Lohner (ALICE), J. Phys. Conf. Ser. 446, 012028 (2013).

[34] W. Cassing and E. L. Bratkovskaya, Nucl. Phys. A 831, 215 (2009).

[35] E. L. Bratkovskaya, W. Cassing, V. P. Konchakovski, and O. Linnyk, Nucl. Phys. A856, 162 (2011).

[36] W. Cassing and S. Juchem, Nucl. Phys. A 665, 377 (2000), ibid. A 672, 417 (2000).

[37] W. Cassing, Eur. Phys. J. ST 168, 3 (2009).

[38] W. Cassing, Nucl. Phys. A795, 70 (2007).

[39] W. Cassing and E. L. Bratkovskaya, Phys. Rept. 308, 65 (1999).

[40] E. L. Bratkovskaya and W. Cassing, Nucl. Phys. A 619, 413 (1997).

[41] E. L. Bratkovskaya, S. M. Kiselev, and G. B. Sharkov, Phys. Rev. C78, 034905 (2008).

[42] A. Peshier, B. Kämpfer, O. P. Pavlenko, and G. Soff, Phys. Rev. D 54, 2399 (1996), P. Levai and U. Heinz, Phys. Rev. C 57, 1879 (1998); A. Peshier, B. Kämpfer, and G. Soff, Phys. Rev. C 61, 045203 (2000), Phys. Rev. D 66, 094003 (2002); M. Bluhm et al., Phys. Rev. C 76, 034901 (2007).

[43] A. Peshier and W. Cassing, Phys. Rev. Lett. 94, 172301 (2005).

[44] W. Cassing and E. L. Bratkovskaya, Phys. Rev. C 78, 034919 (2008).

[45] O. Linnyk, E. Bratkovskaya, J. Manninen, and W. Cassing, J. Phys. Conf. Ser. 312, 012010 (2011).

[46] L. Rauber and W. Cassing, Phys. Rev. D89, 065008 (2014).

[47] V. Konchakovski, W. Cassing, and V. Toneev, J. Phys. G 42, 055106 (2015).

[48] W. Ehehalt and W. Cassing, Nucl. Phys. A 602, 449 (1996).

[49] O. Linnyk, E. L. Bratkovskaya, and W. Cassing, Int. J. Mod. Phys. E17, 1367 (2008).

[50] O. Linnyk, E. L. Bratkovskaya, W. Cassing, and H. Stoecker, Phys. Rev. C76, 041901 (2007).

[51] O. Linnyk et al., Phys. Rev. C84, 054917 (2011).

[52] O. Linnyk, W. Cassing, J. Manninen, E. Bratkovskaya, and C. Ko, Phys. Rev. C85, 024910 (2012).

[53] O. Linnyk et al., Phys. Rev. C87, 014905 (2013).

[54] O. Linnyk, J. Phys. G38, 025105 (2011).

[55] J. Adams et al. (STAR Collaboration), Nucl. Phys. A757, 102 (2005).

[56] K. Adcox et al. (PHENIX Collaboration), Nucl. Phys. A757, 184 (2005).

[57] I. Arsene et al. (BRAHMS Collaboration), Nucl. Phys. A757, 1 (2005).
[58] B. Back, M. Baker, M. Ballintijn, D. Barton, B. Becker, et al., Nucl. Phys. A757, 28 (2005).

[59] S. Turbide, R. Rapp, and C. Gale, Phys. Rev. C69, 014903 (2004).

[60] E. L. Feinberg, Nuovo Cim. A34, 391 (1976).

[61] K. Haglin, C. Gale, and V. Emel'yamnov, Phys. Rev. D47, 973 (1993).

[62] C. Gale and J. Kapusta, Phys. Rev. C 35, 2107 (1987), Phys. Rev. C 38, 2659 (1988), Nucl. Phys. A 495, 423c (1989).

[63] J. Beringer et al. (Particle Data Group), Phys. Rev. D86, 010001 (2012).

[64] F. Low, Phys. Rev. 110, 974 (1958).

[65] W. Liu and R. Rapp, Nucl. Phys. A796, 101 (2007).

[66] H. van Dam and M. Veltman, Nucl. Phys. B22, 397 (1970).

[67] V. Srinivasan et al., Phys. Rev. D12, 681 (1975).

[68] S. Protopopescu et al., Phys. Rev. D7, 1279 (1973).

[69] D. Aston et al., Nucl. Phys. Proc. Suppl. 21, 105 (1991).

[70] K. Haglin, J. I. Kapusta, and C. Gale, Phys. Lett. B224, 433 (1989).

[71] K. L. Haglin, J. Phys. G30, L27 (2004).

[72] L. Landau and I. Pomeranchuk, Dokl. Akad. Nauk Ser. Fiz. 92, 535 (1953).

[73] A. B. Migdal, Phys. Rev. 103, 1811 (1956).

[74] J. Cleymans, V. Goloviznin, and K. Redlich, Phys. Rev. D47, 173 (1993).

[75] J. Cleymans, V. Goloviznin, and K. Redlich, Phys. Rev. D47, 989 (1993).

[76] J. Knoll and R. Lenk, Nucl. Phys. A561, 501 (1993).

[77] W. Cassing, O. Linnyk, T. Steinert, and V. Ozvenchuk, Phys. Rev. Lett. 110, 182301 (2013).

[78] P. B. Arnold, G. D. Moore, and L. G. Yaffe, JHEP 0112, 009 (2001).

[79] O. Linnyk, S. Leupold, and U. Mosel, Phys. Rev. D 71, 034009 (2005), Phys. Rev. D 75 (2007) 014016.

[80] J. Ghiglieri, J. Hong, A. Kurkela, E. Lu, G. D. Moore, and D. Teaney, JHEP 05, 010 (2013).

[81] G. Vujanovic et al., Nucl. Phys. A 932, 230 (2014).

[82] J. Ghiglieri, Nucl. Phys. A 932, 326 (2014).

[83] T. Steinert and W. Cassing, Phys. Rev. C89, 035203 (2014).

[84] Y. Yin, Phys. Rev. C90, 044903 (2014).

[85] ABBHHM (Aachen-Berlin-Bonn-Hamburg-HedielbergMunich Collaboration), Phys. Rev. 175, 1669 (1968).

[86] M. Effenberger, E. Bratkovskaya, and U. Mosel, Phys. Rev. C60, 044614 (1999).

[87] Y. Klopot, A. Oganesian, and O. Teryaev, JETP Lett. 99, 679 (2014).

[88] J. Sakurai, Annals Phys. 11, 1 (1960).

[89] G. Chew, M. Goldberger, F. Low, and Y. Nambu, Phys. Rev. 106, 1345 (1957).

[90] H. Jones and M. Scadron, Annals Phys. 81, 1 (1973).

[91] G. Wolf, G. Batko, W. Cassing, U. Mosel, K. Niita, et al., Nucl. Phys. A517, 615 (1990).

[92] M. Krivoruchenko and A. Faessler, Phys. Rev. D65, 017502 (2002).

[93] M. Zetenyi and G. Wolf, Heavy Ion Phys. 17, 27 (2003).

[94] J. Koch, N. Ohtsuka, and E. Moniz, Annals Phys. 154, 99 (1984).

[95] E. Bratkovskaya, Nucl. Phys. A931, 194 (2014).

[96] M. Aggarwal et al. (WA98 Collaboration), Phys. Rev. Lett. 85, 3595 (2000), and arXiv:nucl-ex/0006007.

[97] K. Dusling and I. Zahed, Phys. Rev. C82, 054909 
(2010).

[98] J. Adam et al. (ALICE), p. arXiv:1509.07324 (2015).

[99] O. Linnyk, E. Bratkovskaya, and W. Cassing, PoS (Baldin ISHEPP XXII), 084 (2015).

[100] A. Adare et al. (PHENIX Collaboration), Phys. Rev. Lett. 107, 252301 (2011).

[101] A. M. Poskanzer and S. Voloshin, Phys. Rev. C58, 1671 (1998).

[102] A. Bilandzic, R. Snellings, and S. Voloshin, Phys. Rev. C83, 044913 (2011).

[103] K. Aamodt et al. (ALICE), Phys. Rev. Lett. 105, 252302 (2010).

[104] M. Aggarwal et al. (WA98 Collaboration), Eur. Phys. J. C41, 287 (2005).

[105] J. Adams et al. (STAR Collaboration), Phys. Rev. C 72, 014904 (2005).

[106] S. Adler et al. (PHENIX Collaboration), Phys. Rev. Lett. 91, 182301 (2003).

[107] A. Adare et al. (PHENIX), p. arXiv:1509.07758 (2015).

[108] L. McLerran, Phys. Part. Nucl. Lett. 8, 673 (2011).
[109] M. Chiu et al., Nucl. Phys. A 900, 16 (2013).

[110] J.-P. Blaizot et al., Nucl. Phys. A 904-905, 829c (2013).

[111] C. Gale et al., Phys. Rev. Lett. 110, 012302 (2013).

[112] B. Bannier (PHENIX Collaboration), Nucl. Phys. A931, 1189 (2014).

[113] V. Voronyuk et al., Phys. Rev. C83, 054911 (2011).

[114] K. Tuchin, Phys. Rev. C91, 014902 (2015), Phys. Rev. C 87 (2012) 024912.

[115] V. Skokov, A. Y. Illarionov, and V. Toneev, Int. J. Mod. Phys. A24, 5925 (2009).

[116] V. Skokov, J. Phys. Conf. Ser. 432, 012021 (2013), G. Basar, D. Kharzeev and V. Skokov, Phys. Rev. Lett. 109 (2012) 202303.

[117] S. Mizuno (PHENIX Collaboration), Nucl. Phys. A931, 686 (2014).

[118] L. Ruan, Nucl. Phys. A931, 185 (2014).

[119] R. Chatterjee, D. K. Srivastava, and T. Renk, Nucl. Phys. A 931, 670 (2014). 